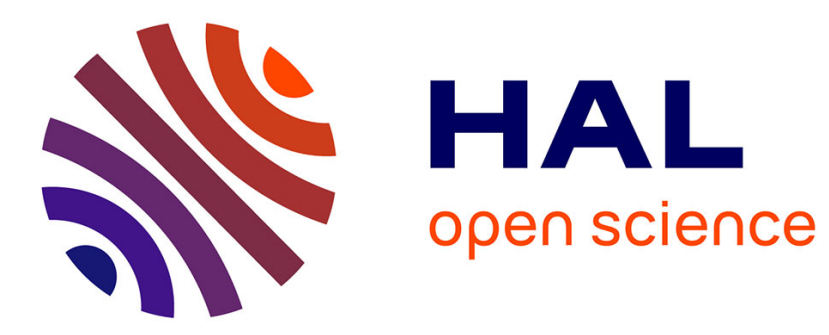

\title{
Influence of a planar boundary on the electric field emitted by a particle shower
}

Daniel García-Fernández, Benoît Revenu, Antony Escudie, Lilian Martin

\section{To cite this version:}

Daniel García-Fernández, Benoît Revenu, Antony Escudie, Lilian Martin. Influence of a planar boundary on the electric field emitted by a particle shower. Phys.Rev.D, 2019, 99 (6), pp.063009. 10.1103/PhysRevD.99.063009 . hal-01952702

\section{HAL Id: hal-01952702 \\ https://hal.science/hal-01952702}

Submitted on 12 May 2020

HAL is a multi-disciplinary open access archive for the deposit and dissemination of scientific research documents, whether they are published or not. The documents may come from teaching and research institutions in France or abroad, or from public or private research centers.
L'archive ouverte pluridisciplinaire HAL, est destinée au dépôt et à la diffusion de documents scientifiques de niveau recherche, publiés ou non, émanant des établissements d'enseignement et de recherche français ou étrangers, des laboratoires publics ou privés. 


\title{
Influence of a planar boundary on the electric field emitted by a particle shower
}

\author{
Daniel García-Fernández, ${ }^{1}$ Benoît Revenu, ${ }^{1,2}$ Antony Escudie, ${ }^{1}$ and Lilian Martin ${ }^{1,2}$ \\ ${ }^{1}$ Subatech, Institut Mines-Télécom Atlantique, CNRS, Université de Nantes, 44307 Nantes, France \\ ${ }^{2}$ Unité Scientifique de Nançay, Observatoire de Paris, CNRS, PSL, UO/OSUC, 18330 Nançay, France
}

(Received 28 November 2018; published 15 March 2019)

\begin{abstract}
The radio detection of cosmic rays consists in the estimation of the properties of a primary cosmic ray by observing the electric field emitted by the extensive air shower (EAS) created when the primary cosmic ray enters the atmosphere. This technique is fully operative nowadays and presents a good degree of maturity. In addition, several projects intend to employ this technique for the detection of neutrinos. In order for the technique to be useful, accurate methods for computing the electric field created by a particle shower in the context of a particular experiment must exist. Although current ground-based radio experiments lie on the air-soil interface and some planned experiments on the South Pole envision antennas near the air-ice interface, most of the analytical approaches and Monte Carlo codes used for calculating the electric field either do not take into account the effect of the boundary or calculate the radiation fields only (direct, reflected and transmitted radiation fields). When the particle shower and the antenna are close to the boundary, compared to the observation wavelength, the far-field approximation breaks down, which is the case for the low-frequency EXTASIS experiment, for instance. We present in this work a new formula for calculating the exact field emitted by a particle track in two semi-infinite media separated by a planar boundary. We also explore the validity of the far-field approximation and make some predictions for EAS using a simple shower model.
\end{abstract}

DOI: 10.1103/PhysRevD.99.063009

\section{INTRODUCTION}

Due to the low flux of cosmic rays with energies above $10^{15} \mathrm{eV}\left(\sim 3 \cdot 10^{-4} \mathrm{~m}^{-2} \mathrm{~min}^{-1}\right)$, high-energy cosmic rays cannot be directly detected and indirect methods must be used. When a cosmic ray enters the atmosphere, it creates an extensive air shower (EAS) that can be detected and whose properties give us information on the primary cosmic ray. The detection of these EAS is carried out nowadays with the help of three main techniques: the surface detection technique, consisting in the measurement of the particles arriving at ground level and, the fluorescence detection, which consists in the collection of the fluorescent light left by the EAS upon its passage through the atmosphere, and finally, the radio detection technique.

The charged particles in the EAS emit an electric field that can be detected using antennas. This is the principle of the radio detection technique. The radio technique is well established nowadays [1], and it presents the capability of measuring the most important properties of cosmic rays, namely, their arrival direction, energy, and composition with urcentainties competitive with respect to the fluorescence detection [2,3]. Radio experiments such as CODALEMA [4], AERA [5], LOFAR [6] or Tunka-Rex [7] detect and analyze cosmic rays on a routinary basis, proving the maturity of the technique at the present time.
Radio detection could be used for detecting neutrinos as well, since neutrinos create a particle shower when they interact within dense media. Initiatives like ARIANNA [8] and ARA [9] try to pave the way for the radio detection of neutrinos on the South Pole. Due to the low cost of the detectors, and if radio detection of neutrinos is feasible, it could be possible to build a radio-based neutrino observatory with an instrumentation volume bigger than the planned new-generation neutrino observatories (IceCubeGen2 [10], KM3NeT 2.0 [11], or GRAND [12]) with a relatively low cost.

In order for the radio technique to be useful, the electric field emitted by the particle showers must be understood. Monte Carlo codes such as SELFAS3 [13,14], ZHAIRES [15] or CoREAS [16] allow the calculation of the emitted field, which enables the reconstruction of the primary cosmic ray upon comparison with the measured data.

Although ZHAIRES can take into account the reflection on the ground for calculating the field received by balloonborne antennas [17], when calculating the field seen by antennas near ground level the effect of the boundary is usually not taken into account by these codes. Only direct fields, as if there were no boundary, are calculated. This is justified by the fact that if the distance between the emitting particles and the receiving antenna is large with respect to the observation wavelength (what is called the far field or 
radiation field), the influence of the boundary can be included either by calculating the reflected field (applying the Fresnel coefficients on the direct field) or knowing the reception pattern of the antenna when the boundary is present, by means of the reciprocity theorem [18,19]. However, this is not true if the observation wavelength is large enough (or, equivalently, the frequency low enough), as such is the case for the EXTASIS experiment $14,20,21]]$, where the observation frequency is less than $5 \mathrm{MHz}(\lambda>60 \mathrm{~m})$. When antennas are located at few hundreds of meters away from the shower core, the exact field emitted by the shower particles hit the ground cannot be obtained by adding the direct and reflected field since geometrical optics no longer apply.

What is the interest, then, of detecting cosmic rays at low frequency? Several experiments, such as Akeno [22] and EAS-TOP [23] have measured an important low-frequency emission from cosmic ray showers (see [24] for a complete review). At low frequencies, the coherence of the shower is expected to be greater, giving rise to an electric field that has more reach than in the standard [20-80] $\mathrm{MHz}$ band. This larger reach at low frequency has been partially confirmed by EXTASIS [20,21]. Another important feature at low frequency is the prediction of a pulse created by the coherent deceleration of the shower particles when the shower particles hit the ground, called the sudden death pulse (SDP) [14]. This pulse has not been seen by EXTASIS, although it might be detected by a lowfrequency experiment at a higher altitude. Besides, in $[14,24,25]$, the SDP has been approximated by the direct emission only, disregarding the effect of the interface. We present in this work a complete approach that includes the effect of the boundary.

Another physical case for which the effect of the boundary on the electric field is important corresponds to the detection of neutrinos in ice, with experiments like the aforementioned ARA and ARIANNA. Whether the antennas are located over the ice or inside the ice, neutrinoinduced showers can cross the air/ice interface, and therefore the antennas will receive field coming from both sides of the boundary. In the far field, the problem can be interpreted as a transition radiation problem and the resulting electric field can be calculated using the far-field formula for a track as a basis to obtain the direct, reflected and transmitted fields [26,27]. Nevertheless, if the antenna and the particle track are close to the surface, the far-field approach is not valid, in principle. It would be desirable, then, to have an exact calculation to employ when the farfield approach breaks down, and also to assess the range of validity of the far-field approach.

In the present work, we calculate the field for a simple dipole and then we increase the level of complexity to a particle track first and then to a shower toy model. We begin deriving in Sec. II the electric field in frequency domain for an electric dipole embedded in a space having two semi-infinite homogeneous media separated by a planar boundary. In Sec. III we use the field of a dipole to construct the exact electric field in frequency domain for a particle track inside the same two semi-infinite media. Then, we prove how it reduces to the formula in [14] if there is no boundary, and as a consequence it can be approximated by the ZHS formula [28] in the far field. After that, we compare the complete formula with a decomposition into direct, reflected, and transmitted fields, both for air/soil and air/ice boundaries. We show as well that the field of a particle inside soil can be neglected. Finally, in Sec. IV we propose a simplified model for an air shower and show that the exact calculation confirms (at least at a theoretical level) the existence of a pulse created by the deceleration of particles at ground level (the SDP), which was already predicted in a less rigorous fashion by ignoring the air/soil interface.

\section{FIELD OF A DIPOLE IN TWO SEMI-INFINITE MEDIA SEPARATED BY A PLANAR BOUNDARY}

Let us assume a three-dimensional space divided by a planar boundary at $z=0$. We define the upper region $(z>0)$ as medium 1 , and the lower region $(z<0)$ as medium 2. Both media are nonmagnetic, that is, their permeability is equal to $\mu_{0}$, the vacuum permeability. Each medium has a relative permittivity $\epsilon_{j r}(j=1,2)$, that can present an imaginary part, indicating absorption. We assume that both media have a conductivity $\sigma_{j}$. Using the $e^{-i \omega t}$ time dependence for the Fourier transform, ${ }^{1}$ the wave number is equal to

$$
k_{j}=\omega \sqrt{\mu_{0}}\left[\epsilon_{0} \epsilon_{j r}+i \frac{\sigma_{j}}{\omega}\right]^{1 / 2},
$$

where $\epsilon_{0}$ corresponds to the vacuum permittivity. We demand that the complex square root in Eq. (1) lies in the upper part of the complex plane, so that the imaginary part of $k_{j}$ is always positive or zero $\left(\Im\left(k_{j}\right) \geq 0\right)$.

Let us now consider a vertical unit dipole vibrating at a given frequency $\omega$, with a current density expressed in the following way:

$$
\mathbf{J}(\mathbf{x}, \omega)=\hat{z} \delta(x) \delta(y) \delta\left(z-z^{\prime}\right) \delta(\omega),
$$

where $z^{\prime}$ is the vertical coordinate of the dipole. Note that we have set the dipole moment equal to $1 \mathrm{~A} \mathrm{~m}$, which will be convenient later for writing the field of a particle track. We can drop the $\delta(\omega)$ factor in Eq. (2) knowing that it must multiply the resulting dipole field (since the dipole vibrates at one given frequency $\omega$ only), and write the new dipole current as

\footnotetext{
${ }^{1}$ Or, equivalently, defining the Fourier transform as $\tilde{f}(\omega)=$ $\int f(t) e^{i \omega t} \mathrm{~d} t$.
} 


$$
\mathbf{J}(\mathbf{x}, \omega)=\hat{z} \delta(x) \delta(y) \delta\left(z-z^{\prime}\right) .
$$

Although one can calculate the electromagnetic potentials or the Hertz potential and derivate to obtain the electric and magnetic fields, for the general case of a dipole in an arbitrary direction it is actually simpler to operate directly with Maxwell's equations expressed as a function of the electric and magnetic fields. The main reason is that the enforcement of the boundary conditions can get a bit cumbersome using the potential formalism, while they are naturally expressed in terms of the fields. We begin by transforming Maxwell's equations to the Fourier space $\left(k_{x}, k_{y}, z, \omega\right)$, with the transform defined as:

$$
\mathbf{A}\left(k_{x}, k_{y}, z, \omega\right)=\int_{-\infty}^{\infty} \mathrm{d} x \mathrm{~d} y \mathrm{~d} t e^{i \omega t-i k_{x} x-i k_{y} y} \mathbf{A}(\mathbf{x}, t) .
$$

With this definition, we transform the horizontal coordinates $(x, y)$ to the Fourier space $\left(k_{x}, k_{y}\right)$ and we switch from time domain to frequency domain as well. The vertical coordinate $z$ remains untouched. Defining the $\tilde{\nabla}$ as

$$
\tilde{\nabla}=i k_{x} \hat{x}+i k_{y} \hat{y}+\frac{\partial}{\partial z} \hat{z}
$$

Maxwell's equations in $\left(k_{x}, k_{y}, z, \omega\right)$ space are formally identical to the standard equations in frequency domain.

$$
\begin{aligned}
& \tilde{\nabla} \times \mathbf{E}_{j}=i \omega \mathbf{B}_{j} \\
& \tilde{\nabla} \times \mathbf{B}_{j}=-\frac{i k_{j}^{2}}{\omega} \mathbf{E}_{j}+\mu_{0} \mathbf{J}
\end{aligned}
$$

with $\mathbf{J}$ given by Eq. (3). The subscript $j$ indicates the upper (1) or lower (2) half-space. Eq. (6) can be decomposed into components and combined in order to obtain solutions given in terms of imaginary exponentials. After that, the resulting electric fields and magnetic fields are required to be continuous. In our case, magnetic fields must be continuous since both media are non-magnetic. The tangential electric field must be continuous as well. The normal component of the electric field must present a discontinuity. If we define the complex electric displacement as the regular electric displacement for a dielectric medium added to a term with the ohmic charge density:

$$
\tilde{\mathbf{D}}=\epsilon \mathbf{E}+\frac{i}{\omega} \sigma \mathbf{E}=\epsilon \mathbf{E}+\frac{i}{\omega} \mathbf{J}_{\mathrm{ohm}} \equiv \tilde{\epsilon} \mathbf{E},
$$

which implies that the divergence of $\tilde{\mathbf{D}}$ depends only on the free, non-ohmic current:

$$
\nabla \cdot \tilde{\mathbf{D}}=\nabla(\epsilon \mathbf{E})+\frac{i}{\omega} \nabla \cdot \mathbf{J}_{\mathrm{ohm}}=\rho_{f}-\rho_{\mathrm{ohm}}=\rho_{f, \mathrm{n}-\mathrm{ohm}},
$$

where $\rho_{f, \mathrm{n}-\mathrm{ohm}}$ is the free, non-ohmic charge density, $\rho_{f}$ is the charge density, and $\rho_{\text {ohm }}$ is the ohmic current. We have used the continuity equation for the ohmic current:

$$
-i \omega \rho_{\mathrm{ohm}}+\nabla \cdot \mathbf{J}_{\mathrm{ohm}}=0
$$

Since the only free, nonohmic current or charge density present in the configuration is the one given by the dipole, Eq. (8) implies that the normal component of the electric field at the boundary, whether in $(\mathbf{x}, \omega)$ space or $\left(k_{x}, k_{y}, z, \omega\right)$ space must satisfy the condition:

$$
\tilde{\epsilon}_{1} E_{1 z}-\left.\tilde{\epsilon}_{2} E_{2 z}\right|_{z=0}=k_{1}^{2} E_{1 z}-\left.k_{2}^{2} E_{2 z}\right|_{z=0}=0 .
$$

The calculation of the fields in each half-space and the enforcing of the boundary conditions can be found in [29]. We will adapt their formulas to our case. Throughout this paper, we will assume that the observer lies in the upper half-space and will calculate the electric field from a source located in the upper and the lower half-space. Of course, if the observer lies in the lower half-space, the fields can be obtained by inverting the $z$ coordinate and swapping the two media.

\section{A. Electric field from a vertical dipole located in the upper half-space}

If both the observer and the dipole are located in medium $1(z>0$, see Fig. 1), the electric field can be written as a sum of direct field and the field created by the boundary. The boundary field can be decomposed into the field created by a perfect image and an integral involving Bessel functions:

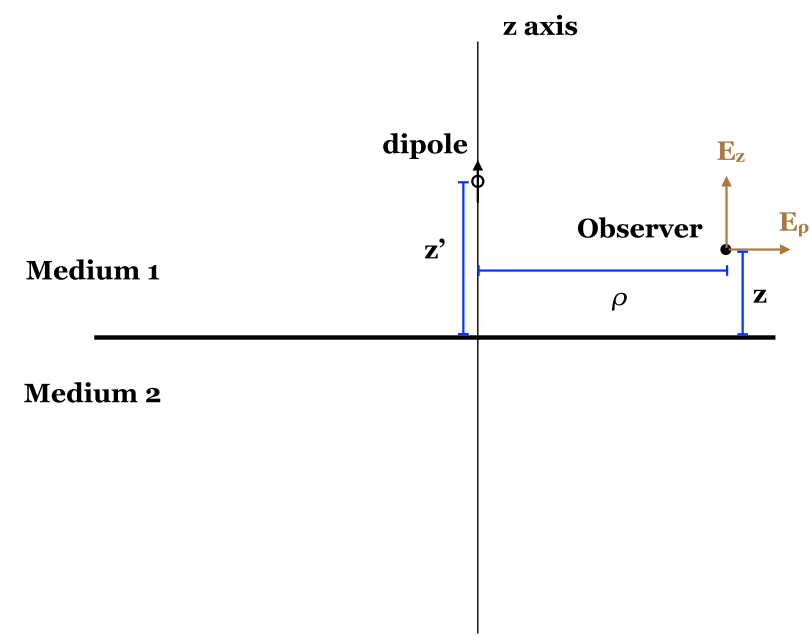

FIG. 1. Sketch of the geometry for the dipole electric field. The figure shows a plane with a fixed azimuthal angle $\varphi$. The dipole is located on the $z$ axis, at $z^{\prime}$. The observer lies at a radial distance $\rho$ and a height $z$. The arrows indicate the radial and vertical components of the electric field. 


$$
\mathbf{E}_{v 1}(\mathbf{x}, \omega)=\mathbf{E}_{\text {direct }}+\mathbf{E}_{\text {boundary }}=\mathbf{E}_{v 1}^{d}+\mathbf{E}_{v 1}^{i m}+\mathbf{E}_{v 1}^{\text {int }} .
$$

We assume the dipole is located at $\left(0,0, z^{\prime} \geq 0\right)$ and the observer at $(\rho \cos \varphi, \rho \sin \varphi, z)$. We define $r_{1}$ as the distance between the dipole and the observer. The radial and vertical components of the direct field can be written as [29]:

$$
\begin{aligned}
E_{1 \rho}^{d}= & -\frac{\omega \mu_{0}}{4 \pi k_{1}^{2}} e^{i k_{1} r_{1}}\left(\frac{i k_{1}^{2}}{r_{1}}-\frac{3 k_{1}}{r_{1}^{2}}-\frac{3 i}{r_{1}^{3}}\right)\left(\frac{\rho}{r_{1}}\right)\left(\frac{z-z^{\prime}}{r_{1}}\right) \\
E_{1 z}^{d}= & \frac{\omega \mu_{0}}{4 \pi k_{1}^{2}} e^{i k_{1} r_{1}}\left[\frac{i k_{1}^{2}}{r_{1}}-\frac{k_{1}}{r_{1}^{2}}-\frac{i}{r_{1}^{3}}\right. \\
& \left.-\left(\frac{z-z^{\prime}}{r_{1}}\right)^{2}\left(\frac{i k_{1}^{2}}{r_{1}}-\frac{3 k_{1}}{r_{1}^{2}}-\frac{3 i}{r_{1}^{3}}\right)\right] .
\end{aligned}
$$

Defining $r_{2}=\sqrt{\rho^{2}+\left(z+z^{\prime}\right)^{2}}$, that is, the distance between the observer and the dipole image at $\left(0,0,-z^{\prime}\right)$, the image field is readily written:

$$
\begin{aligned}
E_{1 \rho}^{i m}= & -\frac{\omega \mu_{0}}{4 \pi k_{1}^{2}} e^{i k_{1} r_{2}}\left(\frac{i k_{1}^{2}}{r_{2}}-\frac{3 k_{1}}{r_{2}^{2}}-\frac{3 i}{r_{2}^{3}}\right)\left(\frac{\rho}{r_{2}}\right)\left(\frac{z+z^{\prime}}{r_{2}}\right) \\
E_{1 z}^{i m}= & \frac{\omega \mu_{0}}{4 \pi k_{1}^{2}} e^{i k_{1} r_{2}}\left[\frac{i k_{1}^{2}}{r_{2}}-\frac{k_{1}}{r_{2}^{2}}-\frac{i}{r_{2}^{3}}\right. \\
& \left.-\left(\frac{z+z^{\prime}}{r_{2}}\right)^{2}\left(\frac{i k_{1}^{2}}{r_{2}}-\frac{3 k_{1}}{r_{2}^{2}}-\frac{3 i}{r_{2}^{3}}\right)\right] .
\end{aligned}
$$

The remaining part of the field is expressed with the help of an integral containing a Bessel function [29]:

$$
\begin{aligned}
& E_{1 \rho}^{\text {int }}=\frac{i \omega \mu_{0} k_{2}^{2}}{2 \pi k_{1}^{2}} \int_{0}^{\infty} \frac{\gamma_{1} e^{i \gamma_{1}\left(z+z^{\prime}\right)}}{N} J_{1}\left(k_{\rho} \rho\right) k_{\rho}^{2} \mathrm{~d} k_{\rho} \\
& E_{1 z}^{\text {int }}=-\frac{\omega \mu_{0} k_{2}^{2}}{2 \pi k_{1}^{2}} \int_{0}^{\infty} \frac{e^{i \gamma_{1}\left(z+z^{\prime}\right)}}{N} J_{0}\left(k_{\rho} \rho\right) k_{\rho}^{3} \mathrm{~d} k_{\rho} .
\end{aligned}
$$

$k_{\rho}$ is an integration variable, $J_{0}$ and $J_{1}$ are the Bessel functions of the first kind of zeroth and first order. $\gamma_{j}$, with $j=1,2$ is defined as

$$
\gamma_{j} \equiv \sqrt{k_{j}^{2}-k_{\rho}^{2}}
$$

and $\mathrm{N}$ is equal to

$$
N \equiv k_{1}^{2} \gamma_{2}+k_{2}^{2} \gamma_{1}
$$

Equations (12)-(14) must be added to obtain the total field, as in Eq. (11). While the direct and image field are expressed in a closed form, the integral in Eq. (14) is not expressible in a closed form in general, and must be computed numerically. Several approximations can be made in order to simplify it. For instance, one can assume [29] that $\left|k_{1}\right| \gg\left|k_{2}\right|, \rho \geq 5 z$, and $\rho \geq 5 z^{\prime}$, but the first of these conditions is not satisfied when medium 1 is the atmosphere. We will show that another useful approximation consists in using the direct field (or the ZHS formula) paired to the Fresnel coefficients when the observer lies in the far field $\left(\Re\left(k_{1}\right) r_{1} \gg 1\right.$ and $\left.\Re\left(k_{1}\right) r_{2} \gg 1\right)$. See Sec. III F.

Due to the oscillatory nature and long tail of the Bessel functions and the imaginary exponential, the integrals in Eq. (14) present a very slow convergence, and partition extrapolation methods have to be used to keep the computation time reasonable. We explain it in Sec. IIE.

\section{B. Electric field from a vertical dipole located in the lower half-space}

Let us place the dipole now at $\left(0,0, z^{\prime}<0\right)$, in the lower half-space, while retaining the observer at $(\rho \cos \varphi, \rho \sin \varphi$, $z>0$ ). The radial and vertical components of the electric field $\mathbf{E}_{2 \rightarrow 1}$ in such a case can be written as [29]:

$$
\begin{aligned}
& E_{2 \rightarrow 1, \rho}=-\frac{i \omega \mu_{0}}{2 \pi} \int_{0}^{\infty} \frac{\gamma_{1} e^{-\gamma_{2} z^{\prime}} e^{i \gamma_{1} z}}{N} J_{1}\left(k_{\rho} \rho\right) k_{\rho}^{2} \mathrm{~d} k_{\rho} \\
& E_{2 \rightarrow 1, z}=\frac{\omega \mu_{0}}{2 \pi} \int_{0}^{\infty} \frac{e^{-i \gamma_{2} z^{\prime}} e^{i \gamma_{1} z}}{N} J_{0}\left(k_{\rho} \rho\right) k_{\rho}^{3} \mathrm{~d} k_{\rho} .
\end{aligned}
$$

Equation (17) corresponds to Eqs. (3.3.13) and (3.3.14) in [29] performing a rotation of $\pi$ around the $x$ axis, which implies the changes $E_{z} \rightarrow-E_{z}, z \rightarrow-z, z^{\prime} \rightarrow-z^{\prime}$, and $\gamma_{1} \rightarrow \gamma_{2}$. As opposed to Eq. (11), Eq. (17) implies that the field from a dipole in the lower half-space can be written as a single integral with no evident closed form, although we will show in Sec. III $F$ that in the far field it is equivalent to the direct field (or ZHS formula) paired with the Fresnel coefficients.

\section{Electric field from a horizontal dipole located in the upper half-space}

Having obtained the field for a vertical dipole, we can solve Maxwell's equations for a horizontal dipole. Instead of Eq. (3), we choose the following current along the $\mathrm{x}$ axis:

$$
\mathbf{J}(\mathbf{x}, \omega)=\hat{x} \delta(x) \delta(y) \delta\left(z-z^{\prime}\right),
$$

which is the current of a horizontal unit dipole. The obtention of the field is analogous to the vertical dipole case, and can be found in [29]. Once again, the field for the horizontal dipole in medium 1 when the observer is in the same medium can be expressed as a sum of three terms.

$$
\mathbf{E}_{h 1}(\mathbf{x}, \omega)=\mathbf{E}_{\text {direct }}+\mathbf{E}_{\text {boundary }}=\mathbf{E}_{h 1}^{d}+\mathbf{E}_{h 1}^{i m}+\mathbf{E}_{h 1}^{\text {int }} .
$$

In this case, however, for an observer at $(\rho \cos \varphi, \rho \sin \varphi, z)$, the azimuthal component of the field is not zero, in general. The direct field is expressed as [29]: 


$$
\begin{aligned}
E_{1 \rho}^{d}= & \frac{\omega \mu_{0}}{4 \pi k_{1}^{2}} \cos \varphi e^{i k_{1} r_{1}}\left[\frac{2 k_{1}}{r_{1}^{2}}+\frac{2 i}{r_{1}^{3}}\right. \\
& \left.+\frac{\left(z-z^{\prime}\right)^{2}}{r_{1}^{2}}\left(\frac{i k_{1}^{2}}{r_{1}}-\frac{3 k_{1}}{r_{1}^{2}}-\frac{3 i}{r_{1}^{3}}\right)\right] \\
E_{1 \varphi}^{d}= & -\frac{\omega \mu_{0}}{4 \pi k_{1}^{2}} \sin \varphi e^{i k_{1} r_{1}}\left(\frac{i k_{1}^{2}}{r_{1}}-\frac{k_{1}}{r_{1}^{2}}-\frac{i}{r_{1}^{3}}\right) \\
E_{1 z}^{d}= & -\frac{i \omega \mu_{0}}{4 \pi k_{1}^{2}} \cos \varphi e^{i k_{1} r_{1}}\left(\frac{\rho}{r_{1}}\right)\left(\frac{z-z^{\prime}}{r_{1}}\right)\left(\frac{k_{1}^{2}}{r_{1}}+\frac{3 i k_{1}}{r_{1}^{2}}-\frac{3}{r_{1}^{3}}\right) .
\end{aligned}
$$

Equation (20) can be obtained from the direct field for the vertical dipole [Eq. (12)] after performing two rotations, which shows that the calculations are consistent (see Appendix). The image field can be written as

$$
\begin{aligned}
E_{1 \rho}^{i m}= & \frac{\omega \mu_{0}}{4 \pi k_{1}^{2}} \cos \varphi e^{i k_{1} r_{2}}\left[\frac{2 k_{1}}{r_{2}^{2}}+\frac{2 i}{r_{2}^{3}}\right. \\
& \left.+\frac{\left(z+z^{\prime}\right)^{2}}{r_{2}^{2}}\left(\frac{i k_{1}^{2}}{r_{2}}-\frac{3 k_{1}}{r_{2}^{2}}-\frac{3 i}{r_{2}^{3}}\right)\right] \\
E_{1 \varphi}^{i m}= & -\frac{\omega \mu_{0}}{4 \pi k_{1}^{2}} \sin \varphi e^{i k_{1} r_{2}}\left(\frac{i k_{1}^{2}}{r_{2}}-\frac{k_{1}}{r_{2}^{2}}-\frac{i}{r_{2}^{3}}\right) \\
E_{1 z}^{i m}= & -\frac{i \omega \mu_{0}}{4 \pi k_{1}^{2}} \cos \varphi e^{i k_{1} r_{2}}\left(\frac{\rho}{r_{2}}\right)\left(\frac{z+z^{\prime}}{r_{2}}\right)\left(\frac{k_{1}^{2}}{r_{2}}+\frac{3 i k_{1}}{r_{2}^{2}}-\frac{3}{r_{2}^{3}}\right) .
\end{aligned}
$$

The integrals for the horizontal case are slightly more complicated than the ones found for the vertical case [29]:

$$
\begin{aligned}
E_{1 \rho}^{\mathrm{int}}= & -\frac{\omega \mu_{0}}{4 \pi k_{1}^{2}} \cos \varphi \int_{0}^{\infty}\left(\frac{\gamma_{1}}{2}(Q-1)\left[J_{0}\left(k_{\rho} \rho\right)-J_{2}\left(k_{\rho} \rho\right)\right]\right. \\
& \left.-\frac{k_{1}^{2}}{2 \gamma_{1}}(P+1)\left[J_{0}\left(k_{\rho} \rho\right)+J_{2}\left(k_{\rho} \rho\right)\right]\right) e^{i \gamma_{1}\left(z+z^{\prime}\right)} k_{\rho} \mathrm{d} k_{\rho} \\
E_{1 \varphi}^{\mathrm{int}}= & \frac{\omega \mu_{0}}{4 \pi k_{1}^{2}} \sin \varphi \int_{0}^{\infty}\left(\frac{\gamma_{1}}{2}(Q-1)\left[J_{0}\left(k_{\rho} \rho\right)+J_{2}\left(k_{\rho} \rho\right)\right]\right. \\
& \left.-\frac{k_{1}^{2}}{2 \gamma_{1}}(P+1)\left[J_{0}\left(k_{\rho} \rho\right)-J_{2}\left(k_{\rho} \rho\right)\right]\right) e^{i \gamma_{1}\left(z+z^{\prime}\right)} k_{\rho} \mathrm{d} k_{\rho} \\
& i \omega \mu_{0} \\
E_{1 z}^{\mathrm{int}}= & \frac{i \pi k_{1}^{2}}{2005} \int_{0}^{\infty}(Q-1) J_{1}\left(k_{\rho} \rho\right) e^{i \gamma_{1}\left(z+z^{\prime}\right)} k_{\rho}^{2} \mathrm{~d} k_{\rho},
\end{aligned}
$$

with

$$
P \equiv \frac{\gamma_{2}-\gamma_{1}}{\gamma_{2}+\gamma_{1}},
$$

and

$$
Q \equiv \frac{k_{1}^{2} \gamma_{2}-k_{2}^{2} \gamma_{1}}{k_{1}^{2} \gamma_{2}+k_{2}^{2} \gamma_{1}} .
$$

\section{Electric field from a horizontal dipole located in the lower half-space}

With the same geometry as in Sec. II B the field from a horizontal dipole in medium 2 seen by an observer in medium 1 is

$$
\begin{aligned}
& E_{2 \rightarrow 1, \rho}=-\frac{\omega \mu_{0}}{4 \pi} \cos \varphi \int_{0}^{\infty}\left(M^{-1}\left[J_{0}\left(k_{\rho} \rho\right)+J_{2}\left(k_{\rho} \rho\right)\right]\right. \\
&\left.+\frac{\gamma_{1} \gamma_{2}}{N}\left[J_{0}\left(k_{\rho} \rho\right)-J_{2}\left(k_{\rho} \rho\right)\right]\right) e^{i\left(-\gamma_{2} z^{\prime}+\gamma_{1} z\right)} k_{\rho}^{2} \mathrm{~d} k_{\rho} \\
& E_{2 \rightarrow 1, \varphi}= \frac{\omega \mu_{0}}{4 \pi} \sin \varphi \int_{0}^{\infty}\left(M^{-1}\left[J_{0}\left(k_{\rho} \rho\right)-J_{2}\left(k_{\rho} \rho\right)\right]\right. \\
&\left.+\frac{\gamma_{1} \gamma_{2}}{N}\left[J_{0}\left(k_{\rho} \rho\right)+J_{2}\left(k_{\rho} \rho\right)\right]\right) e^{i\left(-\gamma_{2} z^{\prime}+\gamma_{1} z\right)} k_{\rho} \mathrm{d} k_{\rho} \\
& i \omega \mu_{0} \\
& E_{2 \rightarrow 1, z} \cos \varphi \int_{0}^{\infty} \frac{\gamma_{2}}{N} J_{1}\left(k_{\rho} \rho\right) e^{i\left(-\gamma_{2} z^{\prime}+\gamma_{1} z\right)} k_{\rho}^{2} \mathrm{~d} k_{\rho} .
\end{aligned}
$$

Equation (17) corresponds to Eqs. (5.4.35), (5.4.36) and (5.4.37) in [29] performing a rotation of $\pi$ around the $x$ axis, which implies the changes $E_{z} \rightarrow-E_{z}, z \rightarrow-z, z^{\prime} \rightarrow$ $-z^{\prime}, E_{\varphi} \rightarrow-E_{\varphi}, \varphi \rightarrow-\varphi$, and $\gamma_{1} \rightarrow \gamma_{2}$. $M$ is defined as:

$$
M=\gamma_{1}+\gamma_{2}
$$

\section{E. Evaluation of Bessel integrals}

Due to the presence of ordinary Bessel functions $J_{n}$, the integrals in Eqs. (14), (17), (22) and (25) contain long oscillating tails that are difficult to evaluate numerically while keeping the computation time reasonable. A slow but easy way to calculate them is to compute the subintegrals in several intervals of the real number line: $\left[0, a_{1}\right],\left[a_{1}, a_{2}\right]$, $\left[a_{2}, a_{3}\right]$, etc., and stop when the sum of the subintegrals reaches convergence with the desired precision. This method is computationally intensive, and the evaluation time of a single integral is of the order of a few seconds.

In order to compute the electric fields in a faster way, we have used an extrapolation method called the partition extrapolation method [30]. Our application of this method goes as follows. We take the intervals $[0, b],[b, 2 b]$, $[2 b, 3 b], \ldots,[(n-1) b, n b]$, and we calculate the integrals of the function in each interval, $I_{1}, I_{2}, \ldots, I_{n}$, using a quadrature adaptative method such as QAG from the GNU Scientific Library (also available via Scipy in Python). Once the value of these integrals is known, the partial sums for each interval are needed, defined as: 


$$
S_{k}=\sum_{i=1}^{k} I_{i}
$$

Let us order these partial sums, $\left[S_{1}, S_{2}, \ldots, S_{n}\right]$. The partition extrapolation method tells us that we can obtain an estimation of the $[0, \infty)$ integral operating on this array by using a triangular scheme. Starting from the array $\left[S_{1}, S_{2}, \ldots, S_{n}\right]$ containing the partial sums, we define another array with $n-1$ elements, and where each element is an average of two contiguous elements of our original array.

$$
\begin{aligned}
& {\left[\frac{1}{2}\left(S_{1}+S_{2}\right), \frac{1}{2}\left(S_{2}+S_{3}\right), \ldots, \frac{1}{2}\left(S_{n-1}+S_{n}\right)\right]} \\
& \quad \equiv\left[S_{1}^{(1)}, S_{2}^{(1)}, \ldots, S_{n-1}^{(1)}\right] .
\end{aligned}
$$

Each iteration can be computed, then, knowing that for the step $k$, the element $i$ is calculated using the elements from the previous step $k-1$,

$$
S_{i}^{(k)}=\frac{1}{2}\left(S_{i}^{(k-1)}+S_{i+1}^{(k-1)}\right) .
$$

After $n-1$ iterations we end up with an array containing a single element, $S_{1}^{(n-1)}$. This element is an estimation for the Bessel integral, and this estimation converges much faster than the brute-force approach [30]. The main idea behind the partition extrapolation method is that the averaging of the partial sums tends to dampen the oscillations of the Bessel function, while converging towards the true value of the integral. The partition extrapolation method allows the use, in general, of different weights for the averaging of the partial sums. However, we have chosen the simple $\frac{1}{2}$ factor because the different weights proposed in [30] resulted in numerical instabilities for our particular integrals.

Depending on the wave number $k$ and the radial distance $\rho$, as well as on the desired precision, a different number of intervals are needed. We will use $b=\pi / \rho$ for the period, while for the number of intervals, we have chosen:

$$
n=\max \left(25,\left\lfloor 16 \frac{\nu}{1 \mathrm{MHz}} \frac{\rho}{1 \mathrm{~km}}\right\rfloor\right) .
$$

$\nu$ represents the linear frequency. For certain observers too close to the dipole, we will use the double of intervals and a period of $b / \sqrt{2}$. With this convention, the relative error of the integral is always less than $10^{-5}$ for the range of frequencies and distances explored throughout this paper.

Once the solution for the field of a dipole is known, we can use it to obtain the complete field created by a particle track.

\section{FIELD OF A PARTICLE TRACK IN TWO SEMI-INFINITE MEDIA SEPARATED BY A PLANAR BOUNDARY}

Let us consider a particle with charge $q$ at rest at the point $\mathbf{x}=\mathbf{x}_{1}$. At a time $t=t_{1}$ the particle is suddenly accelerated and begins to travel in a straight line with a velocity $\mathbf{v}$. Then, at $t=t_{2}$ the particle is abruptly stopped and stays at that place. This trajectory is called a particle track, and it constitutes the building block for particle physics Monte Carlo codes in general, and in particular for the codes that calculate the radio emission from particle showers, such as SELFAS [13], ZHAIRES [15], COREAS [16], or ZHS [28]. The current density of a particle track can be written in time domain as:

$$
\begin{aligned}
\mathbf{J}_{\text {track }}(\mathbf{x}, t)= & q \mathbf{v} \delta^{3}\left(\mathbf{x}^{\prime}-\mathbf{x}_{1}-\mathbf{v}\left(t^{\prime}-t_{1}\right)\right) \\
& \times\left[\Theta\left(t^{\prime}-t_{1}\right)-\Theta\left(t^{\prime}-t_{2}\right)\right],
\end{aligned}
$$

where $\Theta$ is the Heaviside step function. We do not need the charge density for our calculations, since our frequency domain formulas are complete using the current density only, i.e., charge conservation is automatically taken into account if we solve the curl equations in frequency [Eq. (6)], as it has been done for obtaining the dipole fields. Equation (31) can be transformed to frequency domain to yield:

$\mathbf{J}_{\text {track }}(\mathbf{x}, \omega)=q \mathbf{v} \int_{t_{1}}^{t_{2}} \mathrm{~d} t^{\prime} e^{i \omega t^{\prime}} \delta^{3}\left(\mathbf{x}-\mathbf{x}_{1}-\mathbf{v}\left(t^{\prime}-t_{1}\right)\right)$

The electric field created by this current can be calculated as a superposition of the fields for a vertical and a horizontal unit dipole. Since a frame where the track velocity has no $y$ component can always be found, we can assume without loss of generality:

$$
\mathbf{v}=v_{x} \hat{x}+v_{z} \hat{z} \equiv v(\cos \theta \hat{x}+\sin \theta \hat{z}) .
$$

Equations (12), (13), (14), (17), (20), (21), (22), (25) for the fields have been calculated for a dipole located at $\left(0,0, z^{\prime}\right)$, but they are still valid for a dipole at $\mathbf{x}^{\prime}=\left(x^{\prime}, 0, z^{\prime}\right)$ provided the cylindrical coordinate system $(\rho, \varphi, z)$ is correctly centered on the vertical axis that passes through the dipole position. Let $\mathbf{E}_{v(h)}\left(\mathbf{x}, \mathbf{x}^{\prime}, \omega\right)$ be the field created by a vertical (horizontal) unit dipole at $\mathbf{x}^{\prime}=\left(x^{\prime}, 0, z^{\prime}\right)$. The field for a particle track can be expressed as a combination of these fields:

$$
\begin{aligned}
\mathbf{E}_{\text {track }}(\mathbf{x}, \omega)= & \frac{q v}{1 \mathrm{~A} \cdot \mathrm{m}} \int_{t_{1}}^{t_{2}} \mathrm{~d} t^{\prime} e^{i \omega t^{\prime}}\left[\cos \theta \mathbf{E}_{h}\left(\mathbf{x}, \mathbf{x}^{\prime}\left(t^{\prime}\right), \omega\right)\right. \\
& \left.+\sin \theta \mathbf{E}_{v}\left(\mathbf{x}, \mathbf{x}^{\prime}\left(t^{\prime}\right), \omega\right)\right]
\end{aligned}
$$

where $\mathbf{x}^{\prime}\left(t^{\prime}\right)$ is the trajectory of a particle track: 


$$
\mathbf{x}^{\prime}\left(t^{\prime}\right)=\mathbf{x}_{1}+\left(t^{\prime}-t_{1}\right)\left(v_{x} \hat{x}+v_{z} \hat{z}\right) .
$$

We have included a dimensional factor of $1 \mathrm{~A} \cdot \mathrm{m}$, since the unit dipole possesses a moment of the same magnitude. We can define a magnetic field for a particle track in the same way, making the substitution $E \rightarrow B$ in Eq. (34) and taking $\mathbf{B}_{v(h)}$ as the magnetic field from a unit dipole:

$$
\begin{aligned}
\mathbf{B}_{\text {track }}(\mathbf{x}, \omega)= & \frac{q v}{1 \mathrm{~A} \cdot \mathrm{m}} \int_{t_{1}}^{t_{2}} \mathrm{~d} t^{\prime} e^{i \omega t^{\prime}}\left[\cos \theta \mathbf{B}_{h}\left(\mathbf{x}, \mathbf{x}^{\prime}\left(t^{\prime}\right), \omega\right)\right. \\
& \left.+\sin \theta \mathbf{B}_{v}\left(\mathbf{x}, \mathbf{x}^{\prime}\left(t^{\prime}\right), \omega\right)\right]
\end{aligned}
$$

$\mathbf{E}_{v(h)}\left(\mathbf{x}, \mathbf{x}^{\prime}, \omega\right)$ satisfies the following Maxwell's equations:

$$
\begin{aligned}
& \nabla \times \mathbf{E}_{v, h}\left(\mathbf{x}, \mathbf{x}^{\prime}, \omega\right)=i \omega \mathbf{B}_{v, h}\left(\mathbf{x}, \mathbf{x}^{\prime}, \omega\right) \\
& \nabla \times \mathbf{B}_{v, h}\left(\mathbf{x}, \mathbf{x}^{\prime}, \omega\right)=-\frac{i k_{j}^{2}}{\omega} \mathbf{E}_{v, h}\left(\mathbf{x}, \mathbf{x}^{\prime}, \omega\right)+\mu_{0} \mathbf{J}_{v, h}\left(\mathbf{x}, \mathbf{x}^{\prime}, \omega\right),
\end{aligned}
$$

$j=1,2$ and where the currents are given by

$$
\begin{aligned}
& \mathbf{J}_{v}\left(\mathbf{x}, \mathbf{x}^{\prime}, \omega\right)=\hat{z} \delta\left(x-x^{\prime}\right) \delta(y) \delta\left(z-z^{\prime}\right) \\
& \mathbf{J}_{h}\left(\mathbf{x}, \mathbf{x}^{\prime}, \omega\right)=\hat{x} \delta\left(x-x^{\prime}\right) \delta(y) \delta\left(z-z^{\prime}\right)
\end{aligned}
$$

which means that the track current can be expressed by:

$$
\begin{aligned}
\mathbf{J}_{\text {track }}(\mathbf{x}, \omega)= & q \int_{t_{1}}^{t_{2}} \mathrm{~d} t^{\prime} e^{i \omega t^{\prime}}\left(v_{x} \mathbf{J}_{h}\left(\mathbf{x}, \mathbf{x}^{\prime}\left(t^{\prime}\right), \omega\right)\right. \\
& \left.+v_{z} \mathbf{J}_{v}\left(\mathbf{x}, \mathbf{x}^{\prime}\left(t^{\prime}\right), \omega\right)\right),
\end{aligned}
$$

with $\mathbf{x}^{\prime}\left(t^{\prime}\right)$ taken from Eq. (35). Equation (37) implies that the field of a track as defined in Eq. (34) satisfies Maxwell's equations as well and yields the correct current density [Eq. (32)]. The equation for the curl of the electric field is trivially satisfied if we define the magnetic field $\mathbf{B}_{\text {track }}(\mathbf{x}, \omega)$ as in Eq. (36), since the dipole fields satisfy $\nabla \times \mathbf{E}_{v(h)}=i \omega \mathbf{B}_{v(h)}$, and our track field is a linear combination of the dipole fields. As for the curl of the magnetic field,

$$
\begin{aligned}
\nabla \times \mathbf{B}_{\text {track }}(\mathbf{x}, \omega) & =\frac{q}{1 \mathrm{~A} \cdot \mathrm{m}} \int_{t_{1}}^{t_{2}} \mathrm{~d} t^{\prime} e^{i \omega t^{\prime}} \nabla \times\left[v_{x} \mathbf{B}_{h}\left(\mathbf{x}, \mathbf{x}^{\prime}\left(t^{\prime}\right), \omega\right)+v_{z} \mathbf{B}_{v}\left(\mathbf{x}, \mathbf{x}^{\prime}\left(t^{\prime}\right), \omega\right)\right] \\
& =\frac{q}{1 \mathrm{~A} \cdot \mathrm{m}} \int_{t_{1}}^{t_{2}} \mathrm{~d} t^{\prime} e^{i \omega t^{\prime}}\left[-\frac{i k_{j}^{2}}{\omega}\left(v_{x} \mathbf{E}_{h}+v_{z} \mathbf{E}_{v}\right)+\mu_{0}\left(v_{x} \mathbf{J}_{h}+v_{z} \mathbf{J}_{v}\right)\right] \\
& =-\frac{i k_{j}^{2}}{\omega} \mathbf{E}_{\text {track }}+\mu_{0} \mathbf{J}_{\text {track }},
\end{aligned}
$$

where we have used Eq. (39). Equation (40) implies that our solution for the electric field of a particle track [Eq. (34)] satisfies Maxwell's equations, and the source current is precisely the current of a particle track, as intended.

\section{A. Comparison with previous analytical calculations}

With Eqs. (12) and (34), the radial component of the direct electric field created by a vertical track is

$$
\begin{aligned}
E_{\text {track, } 1 \rho}^{d}= & -\frac{q v \omega \mu_{0}}{4 \pi k_{1}^{2}} \int_{t_{1}}^{t_{1}} \mathrm{~d} t^{\prime} e^{i \omega t^{\prime}} e^{i k_{1} r_{1}}\left(\frac{i k_{1}^{2}}{r_{1}}-\frac{3 k_{1}}{r_{1}^{2}}-\frac{3 i}{r_{1}^{3}}\right) \\
& \times\left(\frac{\rho}{r_{1}}\right)\left(\frac{z-z^{\prime}}{r_{1}}\right) .
\end{aligned}
$$

$r_{1}=r_{1}\left(t^{\prime}\right)$, the distance from particle to observer, is now a function of time. Also, $z^{\prime}(t)=z_{1}+v t^{\prime}$. Analogously, for the $z$ component one finds:

$$
\begin{aligned}
E_{\text {track, } 1 z}^{d}= & \frac{q v \omega \mu_{0}}{4 \pi k_{1}^{2}} \int_{t_{1}}^{t_{1}} \mathrm{~d} t^{\prime} e^{i \omega t^{\prime}} e^{i k_{1} r_{1}}\left[\frac{i k_{1}^{2}}{r_{1}}-\frac{k_{1}}{r_{1}^{2}}-\frac{i}{r_{1}^{3}}\right. \\
& \left.-\left(\frac{z-z^{\prime}}{r_{1}}\right)^{2}\left(\frac{i k_{1}^{2}}{r_{1}}-\frac{3 k_{1}}{r_{1}^{2}}-\frac{3 i}{r_{1}^{3}}\right)\right] .
\end{aligned}
$$

Equations (41) and (42) are the same, but with a different notation, as Eqs. (12) and (13) for a vertical track found in [31]. If we make $k_{1}^{2}=\omega^{2} \mu_{0} \epsilon$, and $b=i k_{1}-1 / r_{1}$, we arrive at the same expression, and as a consequence the direct fields of the present work are completely equivalent. This implies as well that they are equivalent to the fields in [25] and that they yield the ZHS formula [28] as a far-field approximation.

\section{B. Evaluation of the field for a single particle track}

After integrand in Eq. (34) is known, the integral from $t_{1}$ to $t_{2}$ must be computed. Equation (34) in conjunction with Eqs. (11) and (19) tells us that if observer and track are in medium 1, the electric field can be written as a superposition of the direct field, the image field, and the integral field from the unit dipole: 


$$
\begin{aligned}
\mathbf{E}_{\text {track }}(\mathbf{x}, \omega) & =\frac{q v}{1 \mathrm{~A} \cdot \mathrm{m}} \int_{t_{1}}^{t_{2}} \mathrm{~d} t^{\prime} e^{i \omega t^{\prime}}\left[\mathbf{E}_{1}^{d}+\mathbf{E}_{1}^{i m}+\mathbf{E}_{1}^{\mathrm{int}}\right] \\
& \equiv \mathbf{E}_{\text {track }}^{d}+\mathbf{E}_{\text {track }}^{\text {im }}+\mathbf{E}_{\text {track }}^{\text {int }},
\end{aligned}
$$

having defined $E_{1}^{d, i m, \text { int }}$ as a combination of the vertical and horizontal unit dipole fields,

$$
\mathbf{E}_{1}^{d, i m, \text { int }} \equiv \cos \theta \mathbf{E}_{h, 1}^{d, i m, \text { int }}+\sin \theta \mathbf{E}_{v, 1}^{d, i m, \text { int }} .
$$

$\mathbf{E}_{v(h), 1}^{d}$ is found in Eq. (12) [Eq. (20)], $\mathbf{E}_{v(h), 1}^{i m}$ in Eq. (13) [Eq. (21)], and $\mathbf{E}_{v(h), 1}^{\mathrm{int}}$ in Eq. (14) [Eq. (22)]. The integral in Eq. (43) can be numerically evaluated with a Riemann sum. However, $\mathbf{E}_{v(h), 1}^{d}$ and $\mathbf{E}_{v(h), 1}^{i m}$ exhibit a $e^{i k r}$ dependence, which allows us to invoke the Fraunhofer approximation. If the parameter

$$
\eta \equiv \frac{k_{1} L^{2}}{r} \sin \theta_{\mathrm{obs}} \ll 1,
$$

where $L$ is the length of the track and $\sin \theta_{\text {obs }}$ the angle between the track velocity and the line joining the track and the observer, we can make the following assumptions:

(1) Fraunhofer approximation for the phase:

$$
k r=k\left|\mathbf{x}-\mathbf{x}^{\prime}\right| \approx k\left[R-v\left(t-t_{0}\right) \cos \theta_{\mathrm{obs}}\right],
$$

where $\mathrm{R}$ is the distance from the observation point to a reference point along the track where the particle is located at a reference time $t_{0}$. For our analysis, the reference point taken will be the middle point of the track.

(2) We assume the distances from track to observer can be taken as constant (except for the one present in the phase), or equivalently

$$
\frac{L}{R} \ll 1
$$

If the above conditions are not fulfilled, we can always subdivide the track until they are. With the above approximations, the integrals can be analytically computed, with the result:

$$
\begin{aligned}
\mathbf{E}_{\text {track }}^{d} \approx & \frac{q v}{1 \mathrm{~A} \cdot \mathrm{m}} e^{i \mathbf{k}_{d} \cdot \mathbf{v} t_{0}}\left[\frac{e^{i\left(\omega-\mathbf{k}_{d} \cdot \mathbf{v}\right) t_{2}}-e^{i\left(\omega-\mathbf{k}_{d} \cdot \mathbf{v}\right) t_{1}}}{i\left(\omega-\mathbf{k}_{d} \cdot \mathbf{v}\right)}\right] \\
& \times \mathbf{E}_{1}^{d}\left(\mathbf{x}, \mathbf{x}_{0}^{\prime}, t_{0}\right)
\end{aligned}
$$

with the track position fixed at:

$$
\mathbf{x}_{0}^{\prime}=\mathbf{x}_{1}+\mathbf{v}\left(t_{0}-t_{1}\right)
$$

$t_{0}$ being:

$$
t_{0}=\frac{t_{1}+t_{2}}{2}
$$

and $\mathbf{k}_{d}$ defined as the wave number times the unit vector from $\mathbf{x}_{0}^{\prime}$ to the observer in $\mathbf{x}$ :

$$
\mathbf{k}_{d}=k_{1} \frac{\mathbf{x}-\mathbf{x}_{0}^{\prime}}{\left|\mathbf{x}-\mathbf{x}_{0}^{\prime}\right|}
$$

Equation (48) reduces to the ZHS formula when dropping all the terms that fall faster than $\frac{1}{r}$ (far-field approximation). The Fraunhofer approximation can be used for the image field as well, but keeping in mind that the expansion has to be made around the image point:

$$
\mathbf{x}_{0, i m}^{\prime}=x_{0} \hat{x}-z_{0} \hat{z}
$$

which is the mirror point of the middle of the track. This modifies as well the definition of the wave number vector:

$$
\mathbf{k}_{i m}=k_{1} \frac{\mathbf{x}-\mathbf{x}_{0, i m}^{\prime}}{\left|\mathbf{x}-\mathbf{x}_{0, i m}^{\prime}\right|}
$$

Other than that, the Fraunhofer approximation for the image field is analogous to Eq. (48):

$$
\begin{aligned}
\mathbf{E}_{\text {track }}^{i m} \approx & \frac{q v}{1 \mathrm{~A} \cdot \mathrm{m}} e^{i \mathbf{k}_{i m} \cdot \mathbf{v} t_{0}}\left[\frac{e^{i\left(\omega-\mathbf{k}_{i m} \cdot \mathbf{v}\right) t_{2}}-e^{i\left(\omega-\mathbf{k}_{i m} \cdot \mathbf{v}\right) t_{1}}}{i\left(\omega-\mathbf{k}_{i m} \cdot \mathbf{v}\right)}\right] \\
& \times \mathbf{E}_{1}^{i m}\left(\mathbf{x}, \mathbf{x}_{0, i m}^{\prime}, t_{0}\right),
\end{aligned}
$$

Equations (48) and (54) can be as accurate as numerical integration, provided the Fraunhofer conditions are satisfied.

Calculating $\mathbf{E}_{\text {track }}^{\text {int }}$, in principle, needs to be done numerically. We can use, for instance, a simple Riemann sum or a Gauss-Legendre quadrature method for an appropriate number of subdivisions of the $\left[t_{1}, t_{2}\right]$ interval. We show in Fig. 2 that the Fraunhofer approximation for the integral is appropriate for the direct and image fields. We have placed a $1.2 \mathrm{~m}$ long electron track whose medium point is at $5 \mathrm{~m}$ from the interface, heading for the ground with a $\pi / 4$ angle with respect to the vector normal to the interface. Medium 1 is air with $\epsilon_{r}=(1.0001)^{2}$ and no conductivity, and medium 2 is an average soil at the Nançay Observatory, where the EXTASIS experiment is located, having $\epsilon_{r}=12$ and a conductivity of $\sigma=5 \mathrm{mS} / \mathrm{m}$. We have placed several observers $20 \mathrm{~m}$ above the boundary and at radial distances of 50,200, and $500 \mathrm{~m}$. The direct and image fields have been calculated using a Riemann sum after dividing the integrand in intervals of $T / 1000$, where $T$ is the period for each frequency, and also with the Fraunhofer approximation by enforcing $\eta<10^{-2}$ [Eq. (45)]. On top of Fig. 2 we have plotted the modules of the electric field for the direct and image fields (points represent the Riemann sum and lines the Fraunhofer approximation), and on the bottom the 

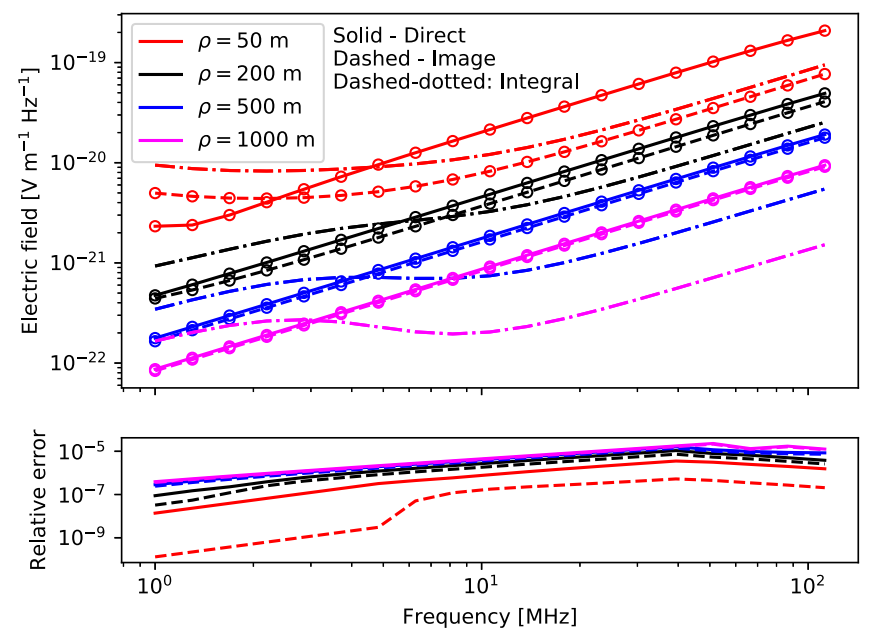

FIG. 2. Top: Electric field as a function of frequency created by a $1.2 \mathrm{~m}$ long electron track traveling at a speed $\sim c$. The middle point of the track is $5 \mathrm{~m}$ above the interface and the electron is traveling towards the boundary with an angle of $\pi / 4$ with respect to the normal. The observers are located at $20 \mathrm{~m}$ above the ground at radial distances of 50 (red circles), 200 (black circles) $500 \mathrm{~m}$ (blue circles), and $1000 \mathrm{~m}$ (magenta circles). Solid lines represent direct fields, dashed lines represent image fields, and dash-dotted lines represent the integral fields. Lines have been calculated using the Fraunhofer approximation and points have been calculated performing a Riemann sum. Bottom: Relative error for the Fraunhofer approximation compared to the Riemann sum. See text for details.

relative error the Fraunhofer approximation. The agreement is better than $<10^{-4}$, which is more than satisfactory for our purposes. We have checked that the complex components of the fields agree, and not only the modules, which is vital to correctly account for interference between particle fields. All of this means that we can use either Eqs. (48) and (54), subdividing when necessary or integrating with whatever numerical method we prefer. The approximation is still valid, as it should, when the track or the observer are far from the boundary.

If the track is in medium 2 and the observer in medium 1 , the transmitted field must be obtained with the help of Eqs. (17) and (25):

$$
\begin{aligned}
& \mathbf{E}_{\text {track }, 2 \rightarrow 1}(\mathbf{x}, \omega) \\
& \quad=\frac{q v}{1 \mathrm{~A} \cdot \mathrm{m}} \int_{t_{1}}^{t_{2}} \mathrm{~d} t^{\prime} e^{i \omega t^{\prime}}\left[\cos \theta \mathbf{E}_{h, 2 \rightarrow 1}+\sin \theta \mathbf{E}_{v, 2 \rightarrow 1}\right] .
\end{aligned}
$$

We will integrate Eq. (55) numerically, as with $\mathbf{E}_{\text {track }}^{\text {int }}$.

\section{Direct, boundary, and total fields}

Although Eq. (43) for the field of a track in medium 1 conveniently divides the electric field in direct, image, and integral fields, it is physically more sound to treat the image and integral fields together as a single entity, since their sum is the field created by the boundary between the two media and that is what must be added to the direct field created by the track. Symbolically,

$$
\mathbf{E}_{\text {track }}(\mathbf{x}, \omega)=\mathbf{E}_{\text {track }}^{d}+\mathbf{E}_{\text {track }}^{i m}+\mathbf{E}_{\text {track }}^{\mathrm{int}} \equiv \mathbf{E}_{\text {track }}^{d}+\mathbf{E}_{\text {track }}^{\text {bound }},
$$

where $\mathbf{E}_{\text {track }}^{\text {bound }}$ represents the field created by the boundary as a response to the particle track. Let us place a $1.2 \mathrm{~m}$ long vertical electron track, reaching the ground and stopping at the boundary. Medium 1 is air and medium 2 is an average soil, as in the previous Section. In Fig. 3, top, we find the
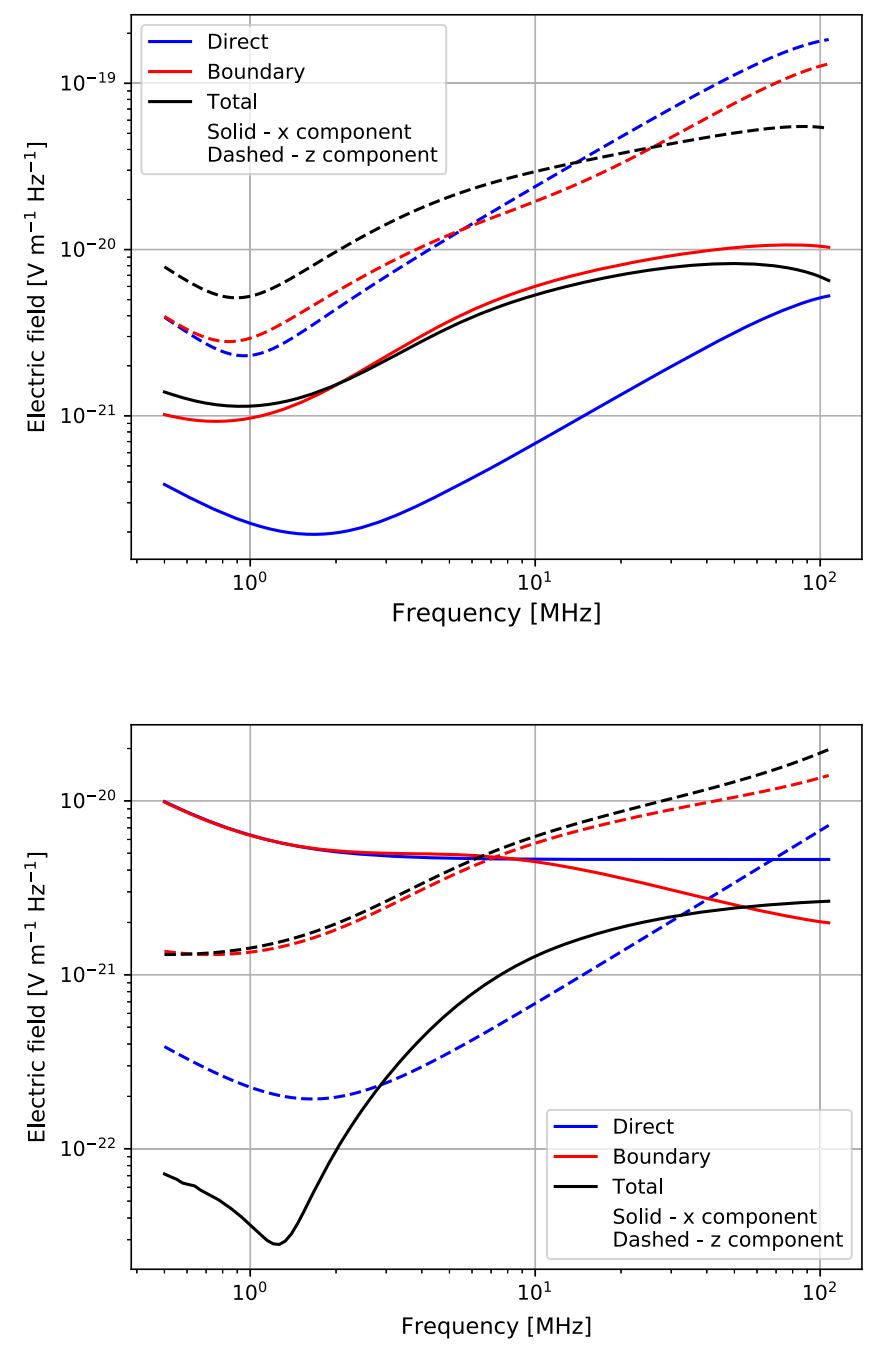

FIG. 3. Top: Components of the electric field as a function of frequency for a $1.2 \mathrm{~m}$ long vertical electron track traveling at a speed $\sim c$. The end point of the track lies at the boundary. The vertical ( $z$, dashed lines) and horizontal ( $x$, solid lines) components are shown. The direct (blue), boundary (red) and total (black) fields are depicted. The observer is located at $\rho=50 \mathrm{~m}$ and $z=2 \mathrm{~m}$ Bottom: Same as top, but for a horizontal track at $z=0.6 \mathrm{~m}$, traveling toward the $+\hat{x}$ direction. 
field seen by an observer located at $\rho=50 \mathrm{~m}$ and $z=2 \mathrm{~m}$. The field created by the boundary is quite important. The $x$ component (solid lines) is completely dominated by the boundary field, while for the $z$ component (dashed lines) there is an interplay between the direct and boundary field, whose interference gives rise to the final form of the total field. In Fig. 3, bottom, we show the field created by a horizontal track at $z=2 \mathrm{~m}$. In this case, the $x$ component (solid lines) is suppressed at low frequencies by the boundary, while the $z$ component (dashed lines) is boosted.

In any case, the conclusion drawn from Fig. 3 is clear. A particle track near the ground creates a non-zero electric field at the position of an observer that lies near ground level also. Moreover, the boundary field plays an important role, which means that for obtaining a rigorous field for particles near a boundary, the direct field is not enough.

We show in Fig. 4 the electric field for a vertical track near the boundary as a function of the radial distance to the observer. At $1 \mathrm{MHz}$ and $5 \mathrm{MHz}$, the boundary amplifies the emission of the track. However, at $50 \mathrm{MHz}$, the direct field and the boundary field interfere destructively, and at several hundreds of meters the field is quite attenuated. Since $50 \mathrm{MHz}$ is near the middle of the bands usually employed by ground-based cosmic ray detection, Fig. 4 implies that we should expect less emission from the shower particles near ground level at standard frequencies. However, at frequencies below $5 \mathrm{MHz}$, where most of the EXTASIS $[14,20,21]$ band is contained, the total field for a track is greater than the direct field, and the coherence at these frequencies between different parts of the shower suggests that the emission from the ground particles of a shower will be amplified thanks to the boundary as well.

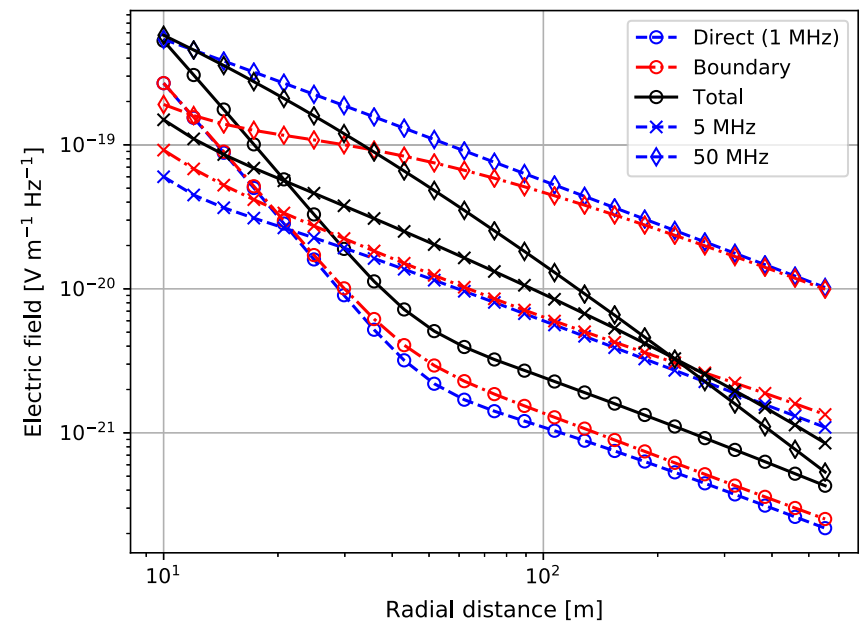

FIG. 4. Module of the electric field as a function of radial observer distance for a $1.2 \mathrm{~m}$ long vertical electron track traveling at a speed $\sim c$. The end point of the track lies at the boundary. The direct (blue, dashed lines), boundary (red, dash-dotted lines) and total (black, solid lines) fields are depicted, for the frequencies of $1 \mathrm{MHz}$ (circles), $5 \mathrm{MHz}$ (crosses) and $50 \mathrm{MHz}$ (diamonds). The observers are located at $z=2 \mathrm{~m}$.

\section{Field from an underground track}

Using Eq. (55) along with Eqs. (17) and (25), we can calculate the field from a particle track immersed in soil, which is useful to assess how the emission in air from the ground particles of the shower relate to the emission in soil, after they reach the ground. We will consider to that effect a vertical electron track that travels $1.2 \mathrm{~m}$ in air with an angle of $\pi / 4$ with respect to the boundary, reaches the ground, and then travels another $0.12 \mathrm{~m}$ in soil in the same direction. We expect the underground particles for a particle shower to be stopped after a few centimeters, so the length of the underground track is reasonable. We show in Fig. 5 the electric fields created by the track, both in air and in soil. We see that the field emitted underground is two orders of magnitude lower than the field emitted in air. We have checked that for vertical tracks, this difference is slightly larger. Therefore, for downward-going tracks, we can ignore the field from the underground track and calculate only the emission when the track is in the atmosphere.

\section{E. Influence of observer height on the electric field from a track}

The field created by the boundary depends on the observer's position with respect to it. As a result, the height of the observer will influence the total field seen by the observer. To illustrate this influence, we have taken a vertical track with $z^{\prime}=100 \mathrm{~m}$ over a ground with $\epsilon_{r}=12$ and $\sigma=5 \mathrm{mS} / \mathrm{m}$, and we have calculated the field for a series of observers located at $\rho=100 \mathrm{~m}$ and variable

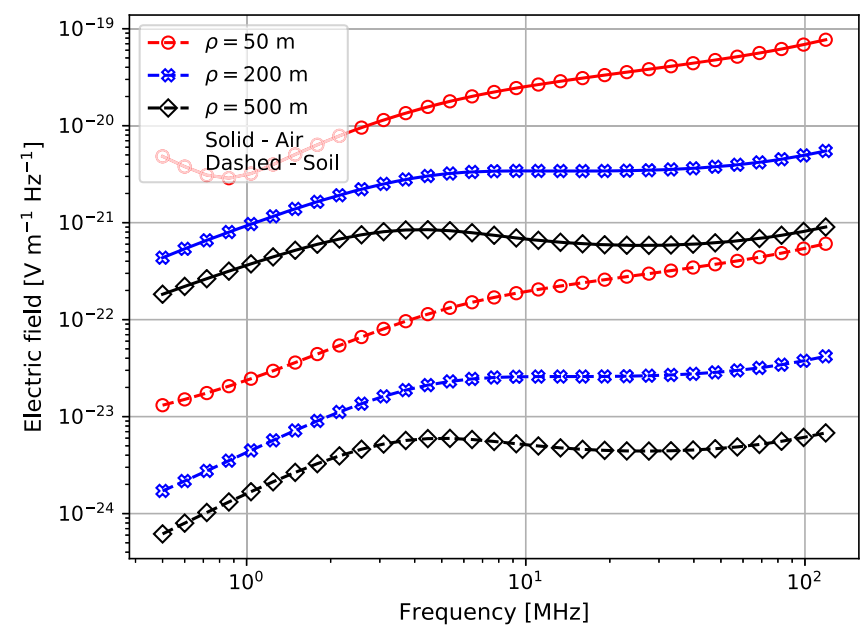

FIG. 5. Module of the electric field as a function of frequency for an electron track traveling at a speed $\sim c$ towards the ground and forming a $\pi / 4$ angle with the interface. The track covers $1.2 \mathrm{~m}$ in air, crosses the boundary, and travels $0.12 \mathrm{~m}$ in an average soil $\left(\epsilon_{r}=12, \sigma=5 \mathrm{mS} / \mathrm{m}\right)$. The emission in air (solid lines) and in soil (dashed lines) for observers at radial distances of 50 (circles), 200 (crosses), and $500 \mathrm{~m}$ (diamonds) are shown. The observers are located at $z=2 \mathrm{~m}$. See text for details. 


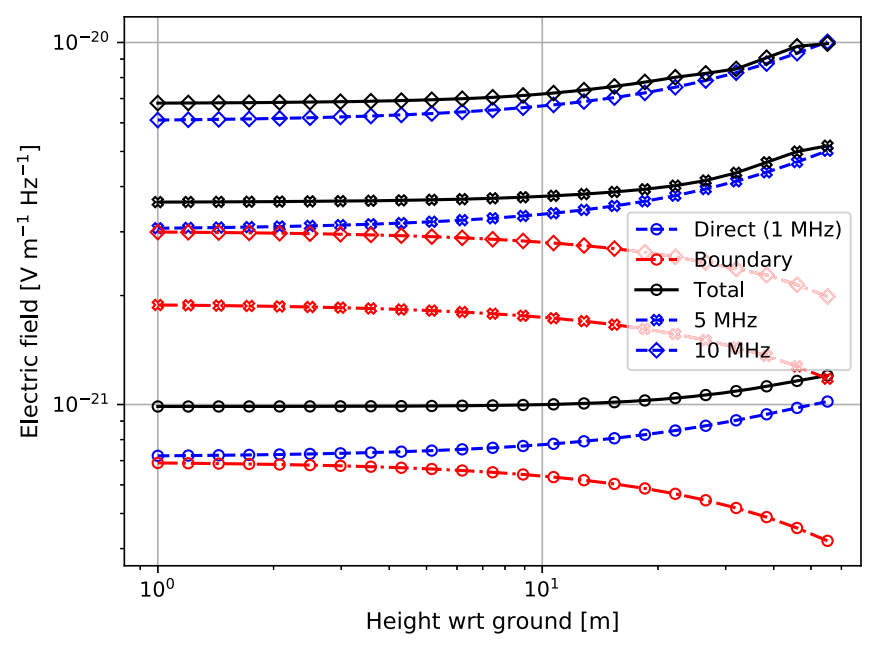

FIG. 6. Module of the electric field as a function of observer height for a $1.2 \mathrm{~m}$ long vertical electron track traveling at a speed $\sim c$. The direct (dashed lines), boundary (dash-dotted lines) and total fields (black lines) for 1 (circles), 5 (crosses), and $10 \mathrm{MHz}$ (diamonds) are shown. The observers are located at $\rho=100 \mathrm{~m}$. See text for details.

height. The results are shown in Fig. 6. We show the fields for 1,5 , and $10 \mathrm{MHz}$. At $1 \mathrm{MHz}$, the dependence of the total field strength with height is less pronounced than at 5 and $10 \mathrm{MHz}$. At $10 \mathrm{MHz}$, the observation wavelength ( 30 m) is about one third of the track's height $(100 \mathrm{~m})$, and since the ground lies near the far-field region of the track, the total field presents an interference pattern similar to the one created by the sum of a direct and a reflected field. We explain in the next section that this is indeed the case.

\section{F. Far field. Decomposition into direct, reflected, and transmitted components}

If either the emitting track or the observer are far away from the boundary compared to the wavelength ( $z$ or $z^{\prime} \ll \lambda$ ), the field should reduce to a sum of direct and reflected fields if the track is in medium 1 . With the help of Fresnel coefficients, and using the direct [Eq. (48)] and image [Eq. (54)] fields, the far-field approximation can be written as:

$$
\mathbf{E}_{\text {track }}^{\mathrm{far}}=\mathbf{E}_{\text {track }}^{\mathrm{d}}+r_{\|} \mathbf{E}_{\text {track }, \|}^{\mathrm{im}}+r_{\perp} \mathbf{E}_{\text {track }, \perp}^{\mathrm{im}} .
$$

The image field has been divided into the polarizations perpendicular and parallel to the reflection plane. The parallel $\left(r_{\|}\right)$and perpendicular $\left(r_{\perp}\right)$ Fresnel reflection coefficients can be written with the help of the following equations, knowing the complex wave numbers $k_{1}$ and $k_{2}$ for each medium and the reflection angle $\theta_{1}$ :

$$
\Re\left(k_{2}\right) \sin \theta_{2}=\Re\left(k_{1}\right) \sin \theta_{1} ; \quad \alpha \equiv \frac{\cos \theta_{2}}{\cos \theta_{1}} ; \quad \beta \equiv \frac{k_{2}}{k_{1}}
$$

$$
r_{\|}=\frac{\alpha-\beta}{\alpha+\beta} ; \quad r_{\perp}=\frac{1-\alpha \beta}{1+\alpha \beta} .
$$

Note that $\theta_{2}$ is the transmitted angle, given by Snell's law. We can compare now the exact calculation [Eq. (43)] with the Fresnel approximation [Eq. (57)]. We will also compare with the ZHS-TR method [26], that combines the ZHS formula and the Fresnel coefficients. If we keep only the leading terms that fall with $\frac{1}{r}$ in Eqs. (12), (20), (13), and (21), we arrive at the same formula. In [31] it is proven that the direct field of a vertical track reduces to the ZHS formula in the far field. Since the field from a horizontal track can be obtained upon rotation (Appendix), the field from a horizontal track reduces as well to the ZHS formula in the far field. The image field, and therefore the reflected field, has the same functional dependence [compare Eqs. (12) and (20) to Eqs. (13) and (21)], which implies that it reduces as well to the ZHS formula in the far field. Adding the Fresnel coefficients to the direct and image ZHS fields $\mathbf{E}_{\text {ZHS }}$, we retrieve the ZHS-TR method:

$$
\mathbf{E}_{\mathrm{ZHS}}^{\mathrm{far}}=\mathbf{E}_{\mathrm{ZHS}}^{\mathrm{d}}+r_{\|} \mathbf{E}_{\mathrm{ZHS}, \|}^{\mathrm{im}}+r_{\perp} \mathbf{E}_{\mathrm{ZHS}, \perp}^{\mathrm{im}} .
$$

We show in Fig. 7 a comparison between the exact calculation [Eq. (43)], the Fresnel approximation [Eq. (57)] and the ZHS-TR formula for a downward going vertical electron track (top) and a horizontal electron track (bottom) at three heights: 10, 100 and $1000 \mathrm{~m}$. We have placed an observer, at a radial distance of $100 \mathrm{~m}$ and $10 \mathrm{~m}$ of height. Figure 7 shows that the exact approach, the Fresnel approximation and the ZHS-TR method agree in the far field. However, for low enough frequencies, neither the Fresnel approximation nor the ZHS-TR method agree with the field predicted by the exact method. When the observation wavelength is 3 times smaller than the distance between the track and the interface, the relative error is around $10 \%$.

$$
\frac{z^{\prime}}{\lambda} \gtrsim 3 \Rightarrow \text { error } \lesssim 10 \%
$$

Putting it in terms of the frequency, for air:

$$
\frac{\nu}{1 \mathrm{MHz}} \frac{z^{\prime}}{1 \mathrm{~km}} \gtrsim 1 \Rightarrow \text { error } \lesssim 10 \%
$$

We can also check that the field emitted by a track in medium 2 can be expressed in the far field as a transmitted field with the help of the Fresnel coefficients. In this case, we are going to assume that medium 1 is air as before, and medium 2 is lossless Antarctic ice with a refractivity of $n_{\text {ice }}=1.78045$. Several observers are placed at a radial distance of $100 \mathrm{~m}$ and heights of 10, 100 and $100 \mathrm{~m}$. This physical configuration is similar to the setup for the 

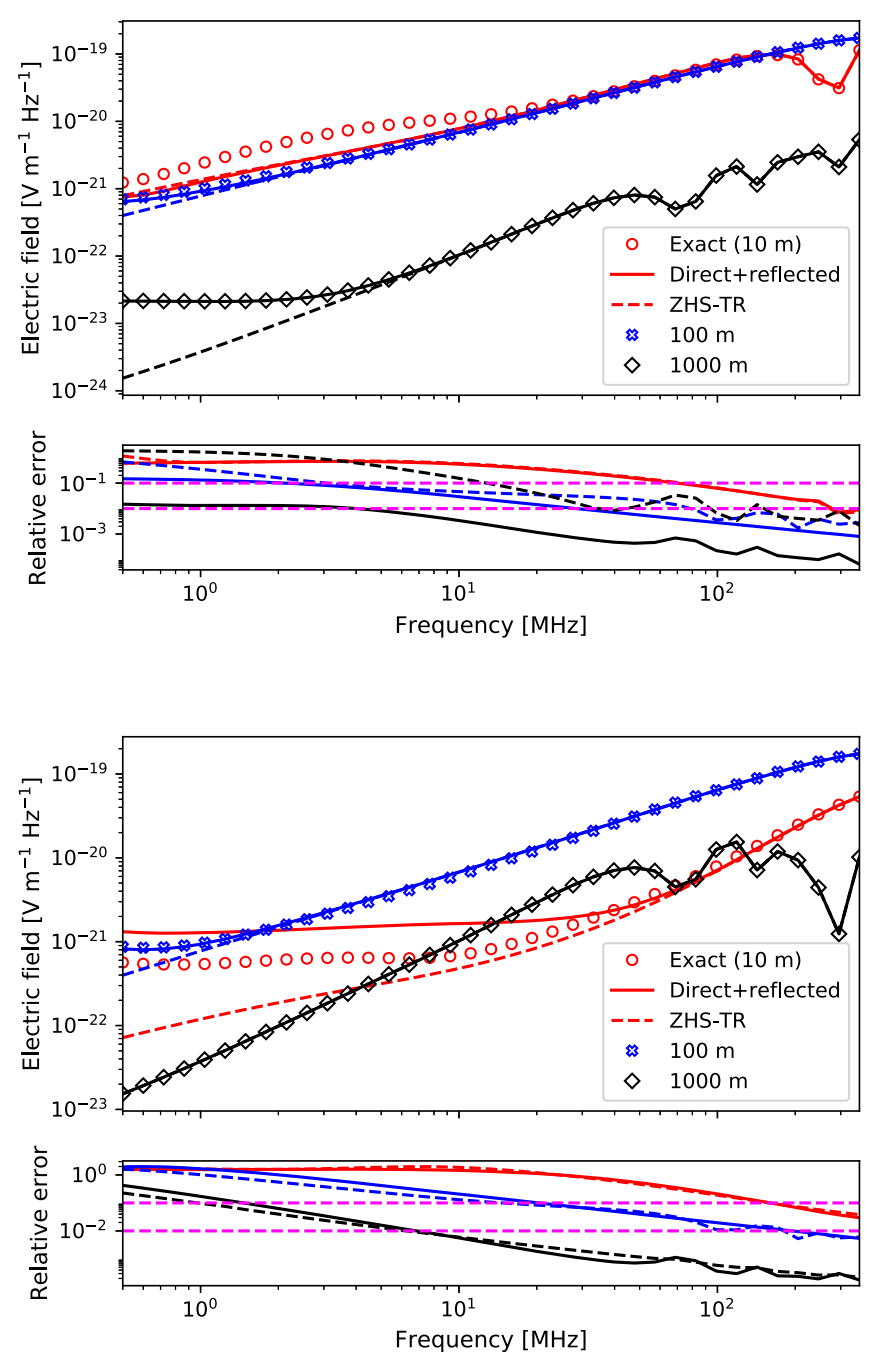

FIG. 7. The four figures are called A, B, C, and D from top to bottom. A: Electric field as a function of frequency created by a $1.2 \mathrm{~m}$ long electron track traveling at a speed $\sim c$. The middle point of the track is located at 10, 100 and $1000 \mathrm{~m}$ above the interface and the track is traveling downwards $(-\hat{z})$ towards the boundary. The observer is located at $\rho=100 \mathrm{~m}$ and $z=10 \mathrm{~m}$. Medium 1 is air and medium 2 is an average soil. The exact fields, the Fresnel (direct plus reflected) approximation and the ZHS-TR formula are shown. B: relative errors of the Fresnel and ZHS-TR approaches with respect to the exact formula. The dashed magenta lines indicate errors of $0.01(1 \%)$ and $0.1(10 \%)$. C and D: Same as A and $\mathrm{B}$, but for a horizontal $(+\hat{x})$ track. See text for details.

ARIANNA [8] antennas that are located on snow, although with larger heights. ${ }^{2}$

The exact field $\mathbf{E}_{\text {track, } \rightarrow 1}$ when the particle is in medium 2 and the observer in medium 1can be approximated in the

\footnotetext{
${ }^{2}$ Note that the present work's formalism can also be applied to study the influence of the air/ice interface when the antennas and the emitted particles are embedded in ice. It suffices to identify medium 1 as ice, place the antennas and particles there, and identify medium 2 as air.
}

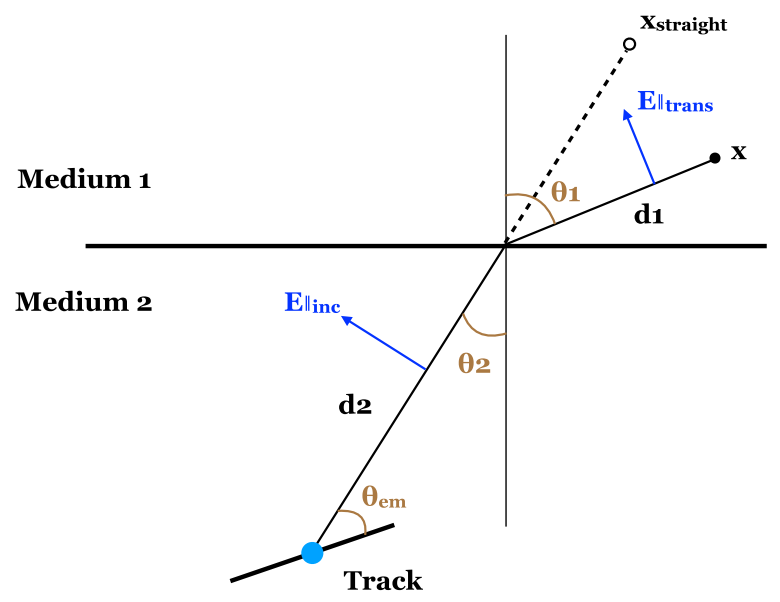

FIG. 8. Sketch explaining the transmission geometry. The figure depicts the refraction plane. The track lies in medium 2, emitting an electromagnetic wave with an emission angle $\theta_{\mathrm{em}}$. The wave field parallel to the refraction plane $\left(\mathbf{E}_{\| \text {inc }}\right)$ arrives at the boundary, and its direction of propagation forms an angle $\theta_{2}$ with the vector normal to the boundary. The field is refracted and enters medium 1 forming a $\theta_{1}$ angle with the normal to the boundary. As a consequence, the transmitted field $\mathbf{E}_{\perp \text { trans }}$ is rotated with respect to the incident field. The transmitted field then arrives at the observer located in $\mathbf{x} \cdot d_{2(1)}$ is the distance traveled by the wave in medium 2(1). $\mathbf{x}_{\text {straight }}$ denotes the apparent position of $\mathbf{x}$ seen from the track position.

far field using the direct field for medium 2 (we suppose that there is no medium 1), the Fresnel transmission coefficients, a rotation matrix, and a phase to correct for the actual path taken by the wave:

$$
\begin{aligned}
& \mathbf{E}_{\text {track }, 2 \rightarrow 1}^{\mathrm{far}}=e^{i\left(k_{1}-k_{2}\right) d_{1}}\left[R\left(\theta_{1}-\theta_{2}\right) T_{\|} \mathbf{E}_{\text {track }, \|}^{\mathrm{d} 2}\left(\mathbf{x}_{\text {straight }}\right)\right. \\
& \left.+T_{\perp} \mathbf{E}_{\text {track }, \perp}^{\mathrm{d} 2}\left(\mathbf{x}_{\text {straight }}\right)\right]
\end{aligned}
$$

$d_{1(2)}$ is the distance traveled by the radiation in medium 1 (2). The phase is there to account for the fact that the direct field in medium 2 does not consider the propagation in medium 1. The direct field is calculated for a point named $\mathbf{x}_{\text {straight }}$, which is the point that lies at a distance $d_{1}+d_{2}$ and along a line that forms a $\theta_{2}$ angle with the normal to the boundary. ${ }^{3}$ This is necessary to ensure that the radiation dependence goes as $1 /\left(d_{1}+d_{2}\right)$ as intended, as well as for calculating the correct emission angle for the radiation which gives the correct polarization. In this case $\theta_{2}$ is the angle that forms the incident wave with the normal and $\theta_{1}$ the refracted angle. We have calculated these angles numerically, since given two points at each side of the interface, there is no general analytical solution for the incident and refracted angles. See Fig. 8 for visual help.

\footnotetext{
${ }^{3} \mathrm{Or}$, equivalently, the apparent location of the observer if we consider the opposite optical path-a ray emitted by the observer that reaches the particle's position.
} 
The parallel $\left(T_{\|}\right)$and perpendicular $\left(T_{\perp}\right)$ transmission coefficients are the Fresnel transmission coefficients multiplied by a correcting factor. Fresnel coefficients are meant to be used for plane waves, but the refraction that takes place when the wave goes from a dense medium to a light medium induces a divergence of the rays, effectively diminishing the electric field. Following [26,27], we write this factor as:

$$
\frac{\mathrm{d} \theta_{2}}{\mathrm{~d} \theta_{1}}=\frac{\Re\left(k_{1}\right) \cos \theta_{1}}{\Re\left(k_{2}\right) \cos \theta_{2}}
$$

which multiplied by the Fresnel transmission coefficients results in:

$$
\begin{aligned}
T_{\|} & =\frac{2 \Re\left(k_{1}\right) \cos \theta_{1}}{\Re\left(k_{1}\right) \cos \theta_{2}+\Re\left(k_{2}\right) \cos \theta_{1}} ; \\
T_{\perp} & =\frac{2 \Re\left(k_{1}\right) \cos \theta_{1}}{\Re\left(k_{2}\right) \cos \theta_{2}+\Re\left(k_{1}\right) \cos \theta_{1}} .
\end{aligned}
$$

The rotation matrix $R\left(\theta_{1}-\theta_{2}\right)$ in Eq. (63) represents a rotation of an angle $\theta_{1}-\theta_{2}$ around the vector normal to the refraction plane, and it is due to the fact that the refraction rotates the polarization of the parallel component of the incident wave. See Fig. 8.

Let us now consider an electron track in ice (medium 2) whose middle point is at $z^{\prime}=-2$. The track is going upwards, forming a $\pi / 4$ angle with the normal to the boundary. We place several observers at $\rho=100 \mathrm{~m}$ and $z=10,100$, and $1000 \mathrm{~m}$. As we can see in Fig. 9, the exact, Fresnel and ZHS-TR calculations agree when frequency is high enough, as it happened with Fig. 7. In this case what changes is the distance from the boundary to the observer (and not the track), but the criterion for the relative error remains unchanged nonetheless. When the $z$ coordinate is 3 times greater than the wavelength, the error is less than $10 \%$.

$$
\frac{z}{\lambda} \gtrsim 3 \Rightarrow \text { error } \lesssim 10 \%
$$

We can conclude that if the particles or the antennas are at a distance from boundary three times larger than the observation wavelength, the decomposition into direct, reflected and transmitted components coupled to the Fresnel coefficients is a good approximation $(\sim 10 \%$ of accuracy) to the exact field. If it is not the case, the exact approach should be used. These results validate the far-field approaches taken in $[26,27]$.

\section{SHOWER TOY MODEL. SUDDEN DEATH PULSE}

After developing and studying the field from a particle track in Sec. III, it would be desirable to implement the
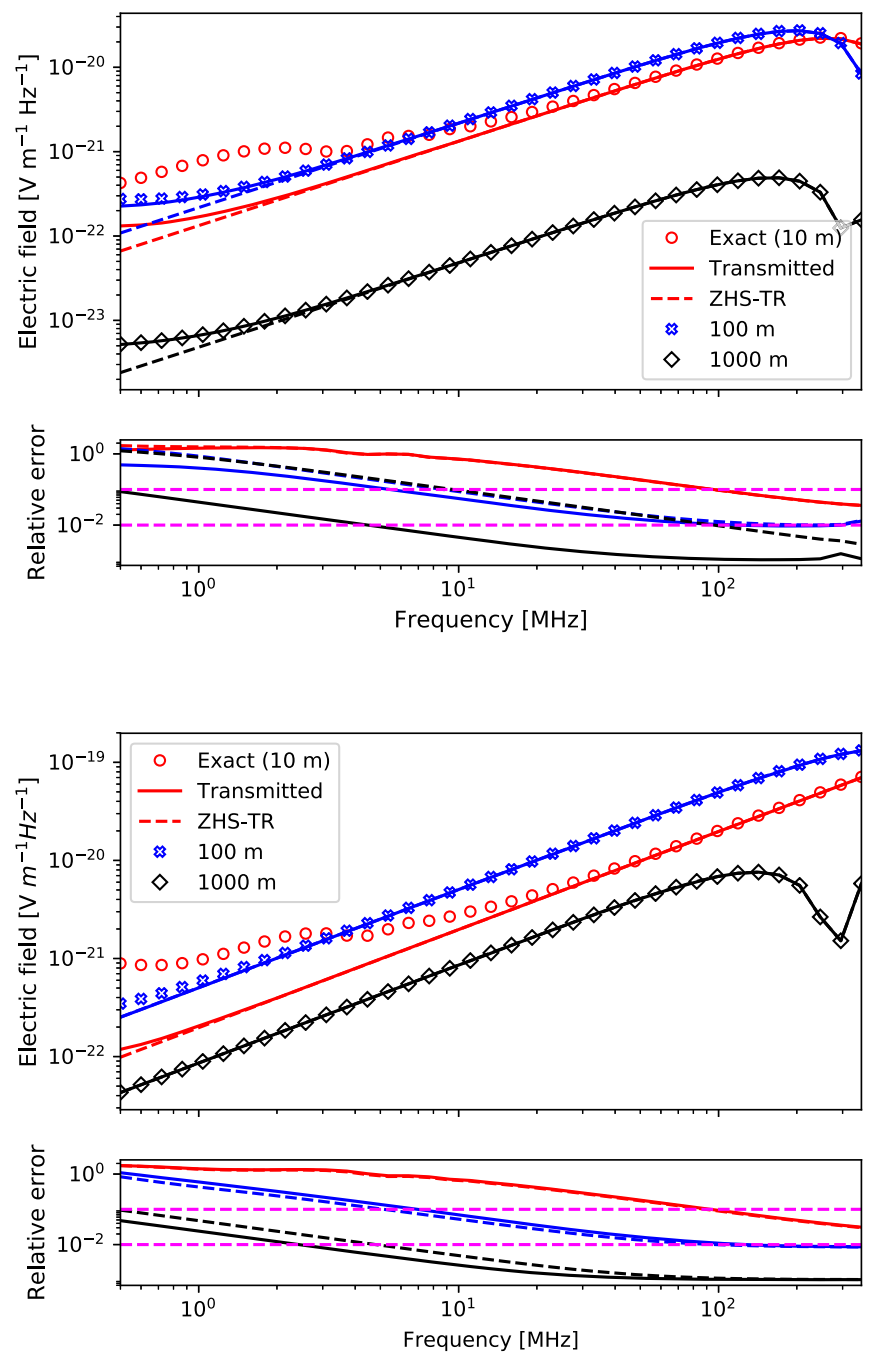

FIG. 9. The four figures are named A, B, C, and D from top to bottom. A: Electric field as a function of frequency created by a $1.2 \mathrm{~m}$ long electron track traveling at a speed $\sim c$. The middle point of the track is located at $z=-2 \mathrm{~m}$, below the interface (medium 2) and the track is traveling towards the boundary with an angle of $\pi / 4$ with respect to the normal. The observers are located at $\rho=100 \mathrm{~m}$ and $z=10 \mathrm{~m}, 100$, and $1000 \mathrm{~m}$. The exact fields, the Fresnel (transmitted) approximation and the ZHS-TR formula are shown. B: relative errors of the Fresnel and ZHS-TR approaches with respect to the exact formula. The dashed magenta line indicates an error of $0.01(1 \%)$. See text for details.

formulas in a Monte Carlo code like SELFAS in order to obtain an accurate prediction for the influence of the ground on the electric field emitted by an EAS. However, the numerical integration of Eqs. (14) and (22) requires an important $\mathrm{CPU}$ time. Even applying the Fresnel approximation when the track is far enough from the ground (as explained in Sec. IIIF), the computation of the field of a track under $20 \mathrm{MHz}$ takes $\sim 10 \mathrm{~s}$ for a single antenna, since each particle trajectory is made up of several tracks, and the field must be known for a set of frequencies. A shower comprised of ten million particles (whether they 
are sampled as in SELFAS or thinned as in ZHAIRES and CoREAS) would take $10^{8} \mathrm{~s}$, more than 3 years.

As an alternative, we will undertake the calculation of the field emitted by a simple model for an EAS. As our goal is primarily to check whether the SDP still exists or not when considering near field effects at the interface, we can consider in our toy model only the contribution of the charge-excess mechanism to the electric field, because the SDP is created by the charge-excess contribution. The aim of this model is to elucidate the modification of the direct field induced by the interface, which constitutes a correction to the direct field prediction shown in [25], and also to verify if the sudden death pulse induced by the coherent deceleration of the shower front is still present.

Let us model the shower as a collection of straight lines stretching from an altitude of $10 \mathrm{~km}$ to the ground level. These lines are positioned at the following radial distances from the shower core: $\rho=\{10,30,50,70,90\} \mathrm{m}$. The choice of these distances is justified because most of the shower particles are contained within the Molière radius, which is $\sim 80 \mathrm{~m}$ near sea level. At higher altitudes, however, the radius is larger because it scales with the inverse of the density, so the model should perform a bit worse for high altitudes. For each one of these distances, we place eight vertical lines at the azimuthal angles $\varphi=\{0, \pi / 4, \pi, \ldots, 7 \pi / 4\}$. In total, our toy model is made up of 40 long lines. Each line is divided into $3 \mathrm{~m}$ long particle tracks whose charge depends on the height $z$ and the radial distance to the shower core:

$$
q_{i}=-0.2 N\left(z_{i}\right) f\left(\rho_{i}\right) A_{i},
$$

$N\left(z_{i}\right)$ is the number of particles at the height $z_{i}$, taken from a Gaisser-Hillas distribution for a $1 \mathrm{EeV}$ shower in our case. The factor -0.2 comes from the negative excess charge in the shower. $f\left(\rho_{i}\right)$ represents the lateral distribution of the shower particles, modeled by a Nishimura-Kamata-Greisen (NKG) function. Since our model effectively transforms the shower into a set of one-dimensional subshowers each one of these subshowers approximate a portion of the shower front with an area given by

$$
A_{i}=\frac{\pi}{4}\left(\left(\rho_{i}+\frac{\Delta \rho}{2}\right)^{2}-\left(\rho_{i}-\frac{\Delta \rho}{2}\right)^{2}\right),
$$

with $\Delta \rho=20 \mathrm{~m}$.

The 80 subshowers start developing at $z=10 \mathrm{~km}$, traveling at $v \sim c$. Every $3 \mathrm{~m}$, the tracks are stopped and another track having a different charge emerges from the stopping point. This process is repeated until the whole shower arrives at ground level, $z_{g}$. The field is calculated for a given observer and several frequencies under $20 \mathrm{MHz}$, where the boundary effects are going to be more prominent. If the height of the track is greater than the wavelength by a factor of 10 , the field is calculated using the Fresnel approximations [Eq. (57)]. Otherwise, the exact field is calculated by means of Eq. (34). Once the fields for the chosen set of frequencies has been calculated, the data are filtered using an eight-order low-pass Butterworth filter (with $\nu_{c}=10 \mathrm{MHz}$ as critical frequency) zero-padded, and transformed to time domain to obtain the time trace of the electric field.

We must stress that using this simple model we are only calculating the excess charge emission from the particle shower, but this is fine because we are interested only in knowing what happens with the SDP emission near the boundary. The main contribution to the electric field emitted by a real air shower comes from the geomagnetic effect, although the ratio of the geomagnetic and excess charge emission mechanisms depends on the shower arrival direction and the local geomagnetic field. The charge excess mechanism typically represents $\sim 15 \%-20 \%$ of the electric field of a real shower.

We can find in Fig. 10 the electric field in time domain given by our model for a vertical shower. Ground (or emission) altitude is $z_{g}=0 \mathrm{~m}$, and we have placed two observers at 300 and $500 \mathrm{~m}$ of radial distance and at $9 \mathrm{~m}$ of height, which is the height of the antennas used for the EXTASIS experiment. Medium 1 is air, with an $\epsilon_{r}=$ $(1.0001)^{2}$ and no conductivity, and medium 2 is again an average soil with $\epsilon_{r}=12$ and $\sigma=5 \mathrm{mS} / \mathrm{m}$. The direct [Eq. (48), dashed black lines], direct plus reflected [Fresnel approximation, Eq. (57), dash-dotted blue lines] and exact [Eq. (34), solid red lines] are plotted. The left part of the trace is due to the shower maximum. Since the maximum is located at several kilometers of altitude, the Fresnel

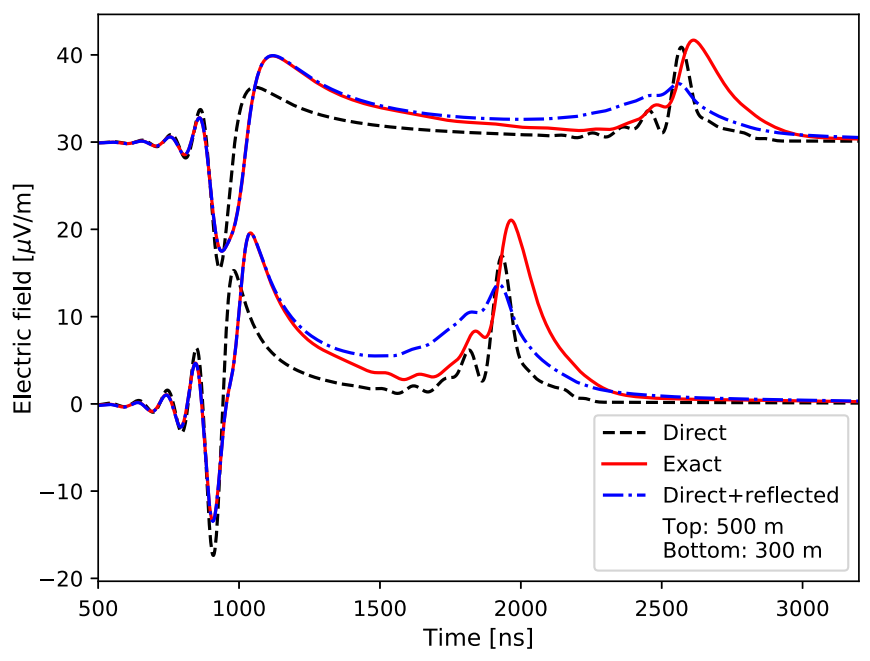

FIG. 10. Electric field in time domain created by our model shower traveling at a speed $\sim c$. Medium 1 is air and medium 2 is an average soil. The observers are located at $\rho=300$ (lower curves) and $500 \mathrm{~m}$ (upper curves, electric field offset by $30 \mu \mathrm{V} / \mathrm{m}$ ) and $z=9 \mathrm{~m}$. The exact fields (solid red line), the Fresnel approximation (direct + reflected, dash-dotted line) and the direct (dashed black lines) are shown. See text for details. 
approximation and the exact calculation agree on the emission from the shower maximum. As time passes and the shower develops, the particles get closer to the ground and the Fresnel approximation ceases to be valid. When the particles stop at ground level, their sudden deceleration creates the second peak that can be seen around $2000 \mathrm{~ns}$ for the observer at $300 \mathrm{~m}$ (bottom) and aroud $2500 \mathrm{~ns}$ for the observer at $500 \mathrm{~m}$ (top). This is the sudden death pulse (SDP) already discussed in [14,25], although in those references only the direct emission had been computed. The present work's approach shows that although the exact calculation and the direct emission differ, the SDP is however still present and presents similar properties to the ones outlined by the direct calculation:

(1) The amplitudes and durations of the exact and direct SDPs are of the same order of magnitude.

(2) The delay between the principal pulse and the SDP is directly proportional to the distance from the shower core to the observer (see Fig. 13, bottom), as with the direct case.

(3) The amplitude of the SDP falls with the inverse of the distance from shower core to observer (see Fig. 13, top), as it happened with the direct case.

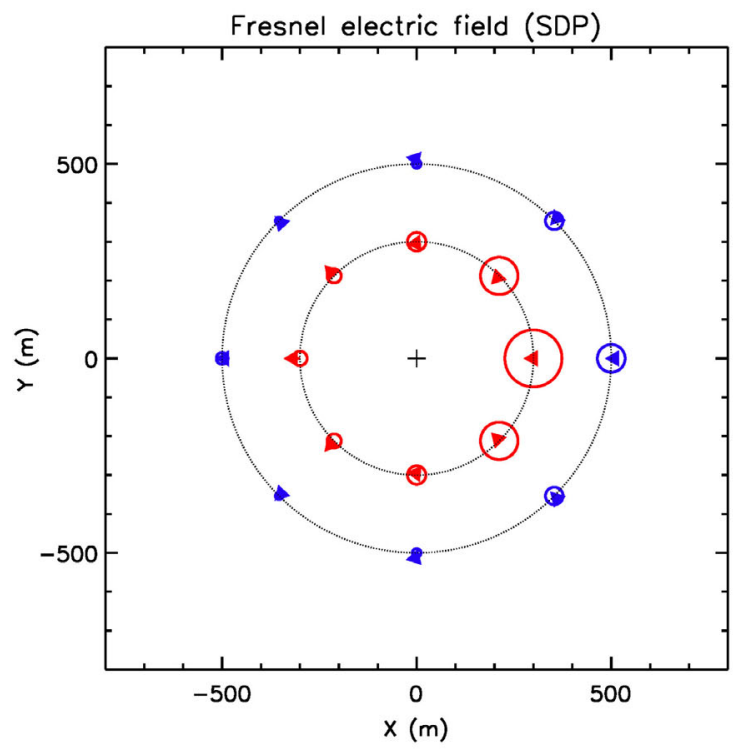

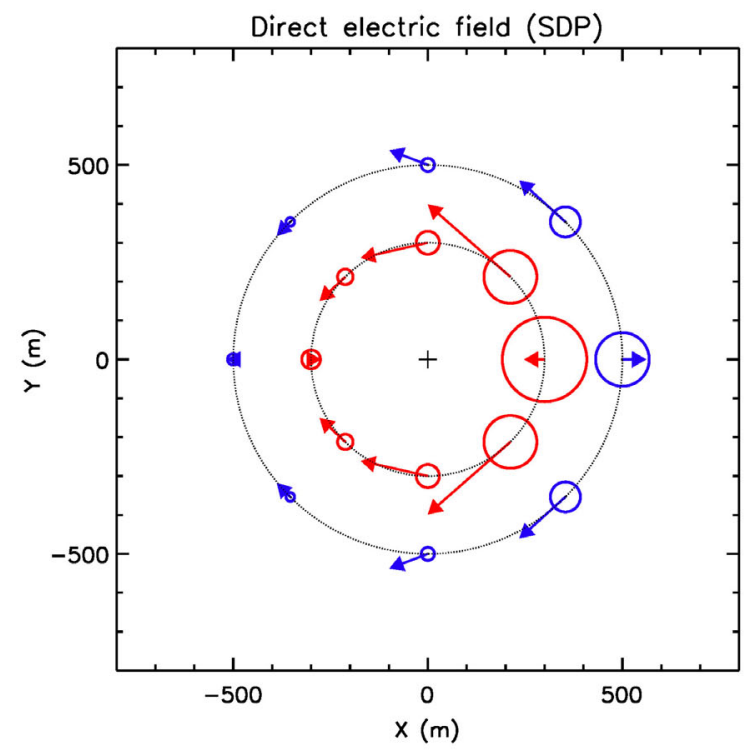

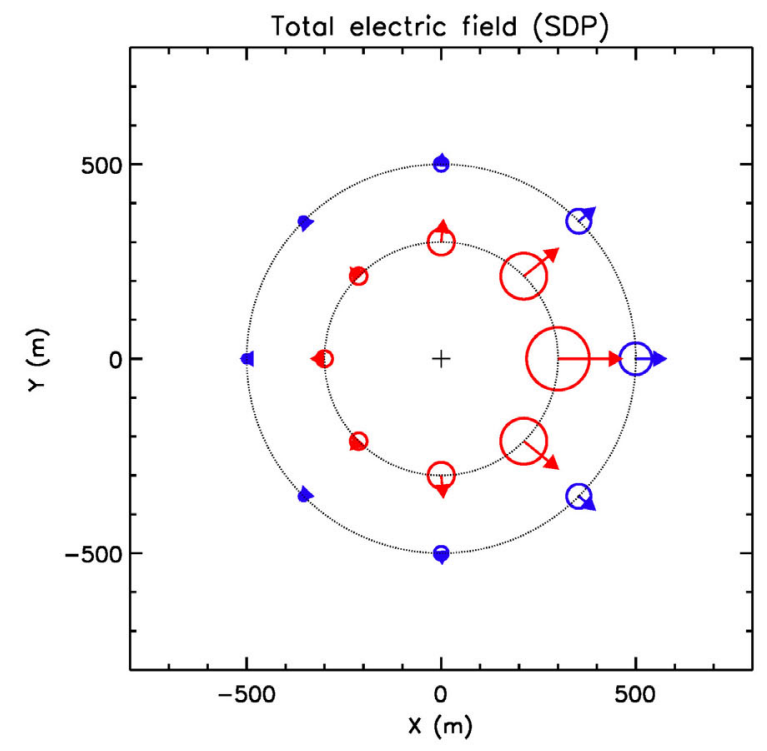

FIG. 11. Polarization map of the SDP emitted by a $30^{\circ}$ zenith angle shower coming from the $-\hat{y}$ direction $\left(\varphi=180^{\circ}\right)$, with the core at $(0,0)$. Black points indicate the position of the antennas, located on two rings $300 \mathrm{~m}$ and $500 \mathrm{~m}$ away from the shower core. Arrows indicate the direction and amplitude of the horizontal polarization while the radius of the circles indicate the amplitude of the vertical polarization (pointing always on the $\hat{z}$ direction). Ground altitude is $1400 \mathrm{~m}$. Top left: direct field. Top right: direct + reflected fields (Fresnel approximation). Bottom: total exact field. See text for details. 
One interesting feature found in Fig. 10 is the different time at with the maximum of the SDP occurs for the direct (or direct + reflected) and the exact calculation. The exact maximum arrives slightly later than the direct or direct + reflected maximum, and the reason for that is the surface wave (or lateral wave) that propagates along the boundary and therefore has a longer path than the direct and reflected waves.

When the shower is vertical, the SDP presents vertical polarization only. This is related to the fact that, in our model, the deceleration at ground level lies along the $\hat{z}$ direction. To know if other polarizations are possible, we have simulated a $\theta=30^{\circ}$ zenith angle shower coming from the azimuth $\varphi=180^{\circ}$. We show a polarization map for the direct, direct + reflected and total exact fields in Fig. 11, where the arrows indicate the horizontal polarization and the size of the circles represent the vertical polarization. When comparing the direct vertical component (Fig. 11, top left) with the exact one (Fig. 11, bottom), we see that the surface wave enhances the total vertical component. However, the horizontal polarization is slightly suppressed and its direction differs from that obtained for the direct field. We have checked that the Fresnel approximation (direct + reflected) does not suffice for understanding this behavior, as depicted in Fig. 11 (top right). Our calculations show that the amplitude of the vertical component of the SDP is similar to the direct field, and therefore the vertical polarization of the SDP for a real shower should be similar to the ones in [14]. However, the horizontal (east-west and north-south) polarizations in [14] for the SDP do not constitute a good approximation, in principle.

We show in Fig. 12 the influence of the type of ground on the total field. The ground altitude is fixed at $z_{g}=0 \mathrm{~m}$. We have chosen an average soil used for the EXTASIS antennas simulations $\left(\epsilon_{r}=12, \sigma=5 \mathrm{mS} / \mathrm{m}\right)$, the soil present at the AERA experiment [32] both for dry $\left(\epsilon_{r}=2, \sigma=1 \mathrm{mS} / \mathrm{m}\right)$ and damp conditions $\left(\epsilon_{r}=10\right.$, $\sigma=1 \mathrm{mS} / \mathrm{m})$, and seawater [33] $\left(\epsilon_{r}=70, \sigma=5 \mathrm{~S} / \mathrm{m}\right)$. As it was already expected, since the emission from the shower maximum can be approximated by a direct and a reflected component, and the reflected component depends on the properties of the soil, the amplitude of the principal pulse (left) varies with the type of soil used. In fact, choosing between a dry or damp ground changes the field more than $10 \%$, which reminds the importance of knowing the ground and its influence on the measuring antennas. The SDP (right pulse) is also heavily affected by the type of ground, since the properties of the surface wave are related to the properties of the ground. A higher conductivity results in a larger surface wave, while the influence of the permittivity seems to be more complicated.

Although the resulting SDP fields present an important component that is created by the surface wave, which is a nonradiative type of field since it does not carry energy

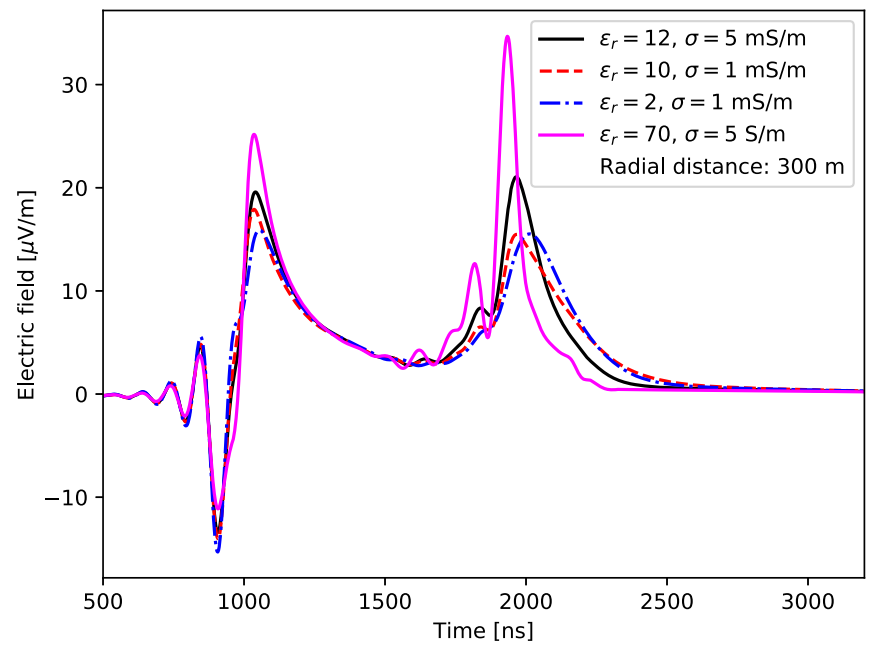

FIG. 12. Vertical component of the total exact electric field predicted by our shower model for a vertical shower. Different grounds have been used: average soil at EXTASIS (solid black line, $\epsilon_{r}=12, \sigma=5 \mathrm{mS} / \mathrm{m}$ ), damp AERA soil (dashed red line, $\epsilon_{r}=10, \sigma=1 \mathrm{mS} / \mathrm{m}$ ), dry AERA soil (dash-dotted blue line, $\epsilon_{r}=2, \sigma=1 \mathrm{mS} / \mathrm{m}$ ) and seawater (solid magenta line, $\epsilon_{r}=70$, $\sigma=5 \mathrm{~S} / \mathrm{m}$ ) See text for details.

towards the infinity (it vanishes at large distances from the interface, where the radiative direct and reflected components dominate), we can see in Fig. 13, top, that the SDP amplitudes for a vertical shower according to the model fall with the inverse of the distance to the shower core, as if it were a pure radiation field. This result is in agreement with the direct field calculation in [14]. We retrieve as well that the SDP amplitude increases when the ground altitude is higher, due to the larger number of particles arriving at the ground.

We show in Fig. 13, bottom, the arrival time of the SDP as a function of distance. $t=0$ is the time the shower reaches ground. The data for 1400 and $2500 \mathrm{~m}$ of altitude have been offset for clarity. The arrival times grow linearly with distance as evidenced by the superposed linear fits. The inverses of the fit slopes are 0.2966, 0.2961 and $0.2958 \mathrm{~m} \mathrm{~ns}^{-1}$ for 0,1400 , and $2500 \mathrm{~m}$ of altitude respectively. The speed of light in air is $\sim 0.2998 \mathrm{~m} \mathrm{~ns}^{-1}$, which is slightly larger, reflecting the fact that the surface wave travels close to the boundary and therefore has a lower effective speed than the direct and reflected components. However, there is a linear relation between the SDP arrival time computed using the surface wave and the distance to the shower core, as it was the case for the direct field only [14].

Although this simple model does not consider geomagnetic effects, we expect that for pure geomagnetic emission the effect of the boundary on the principal pulse will be well approximated by the far-field calculation, as it is the case for the excess charge effect. However, the SDP is created precisely by the excess of negative charge that is 

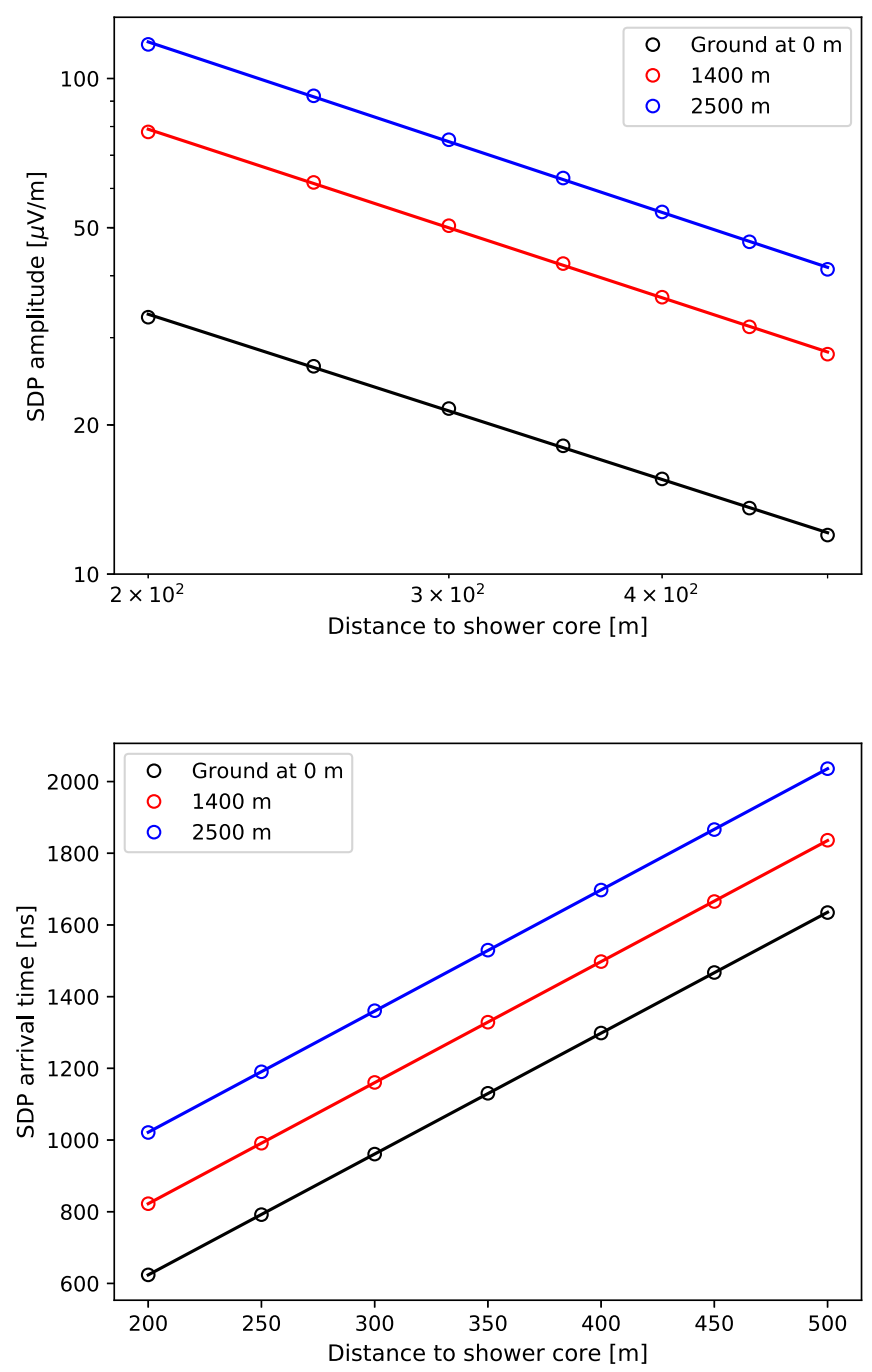

FIG. 13. Top: Amplitude of the SDP maximum created by a vertical shower as function of the distance to the shower core. The observers are located at a height of $2 \mathrm{~m}$ above the ground. Ground altitudes are $0 \mathrm{~m}$ (lower curve), $1400 \mathrm{~m}$ (middle curve) and $2500 \mathrm{~m}$ (upper curve). Points represent the SDP amplitudes and lines show a $1 / R$ fit to the data, with $R$ being the distance from the observer to the shower core. Medium 1 is air and medium 2 is an average soil. Bottom: Same setup as top, but now the $y$ axis shows the arrival time of the SDP maximum, zero being the time when the shower reaches the ground (data for $1400 \mathrm{~m}$ and $2500 \mathrm{~m}$ are offset by 200 and $400 \mathrm{~ns}$, respectively). Lines indicate a linear fit for the arrival time. See text for details.

suddenly decelerated, meaning that for a purely geomagnetic emission there would be no SDP.

\section{SUMMARY AND CONCLUSIONS}

Since the field of a dipole can be used to calculate the field created by more complex configurations, we have discussed the exact frequency-domain electric field created by a unit dipole in two semi-infinite media separated by a planar boundary. If the dipole and the observer are in the same medium, the field can be separated in three fields: a direct field, an ideal image field, and an integral that contains information about the lateral wave created by the boundary. If the observer and the dipole lie in different media, the whole field can be expressed with a single integral that yields the field that passes through the boundary. In both cases, the integrals have to be numerically evaluated, in general. The integrals contain Bessel functions with long oscillating tails that present a very low convergence. To keep the computation time reasonable, we have chosen the partition extrapolation method as our method of integration.

Then, we have presented a frequency-domain equation produced by a particle track that takes into account the effect of the boundary on the electric field at all frequencies. This field has been obtained by integrating the solution for the electric field of a dipole, previously obtained. As with the dipole case, the track field can be divided into direct, image, and integral fields if the observer and the track lie in the same medium [Eq. (43)]. However, in this case the three partial fields have to be numerically integrated, in general. We have shown that the Fraunhofer approximation for integrating the direct and image fields is a good approximation. In the far field, where either the track or the observer are far away from the boundary, the track field is expressible by the sum of a direct and reflected field (using Fresnel coefficients). Our formula is also equivalent to the ZHS-TR formalism [26] in the far field. We have also obtained a solution for the case when the observer and the track are in different media [Eq. (55)], which reduces in the far field to a transmitted wave calculated using a modified Fresnel coefficient as in $[26,27]$. The transmitted field calculated in the present work is also consistent with ZHSTR. As a rule of thumb, when either the distance from track to boundary or the distance from observer to boundary is 3 times greater than the observation wavelength, the relative error of the far-field approximation is less than $10 \%$.

We have studied the spectra for a track in air near a soil ground. For frequencies under $10 \mathrm{MHz}$, which is the domain of the EXTASIS experiment, the boundary creates a surface wave that interferes constructively with the direct field. The behavior of these spectra with radial distance shows that, under $10 \mathrm{MHz}$, the contribution of the surface wave is quite relevant up to radial distances of several hundreds of meters. We have also calculated the field from a short underground track seen by an observer in the atmosphere and found that the underground field is two orders of magnitude smaller than the field emitted by a typical particle track traveling in air near the boundary, which implies that the underground particles can be ignored.

Since the computation time for the exact electric field from a realistic shower simulated with a Monte Carlo code seems unmanageable, we have proposed a simple model for a EAS. We have calculated the exact field under $10 \mathrm{MHz}$ for a set of one-dimensional subshowers presenting a 
Gaisser-Hillas longitudinal profile together with a NKG lateral distribution. The results show that the emission coming from the shower maximum is not modified by the boundary field, but when the shower is near the ground the influence of the boundary is rather important. The exact calculation confirms that a sudden death pulse (SDP) is created when the shower abruptly stops at ground level, which had already been obtained in a less rigorous way in [14] using the direct field only. The order of magnitude of the vertical component of the SDP predicted by the exact calculation is the same as that predicted by the exact calculation, which means that the direct emission is a decent approximation for the vertical field and the calculations of [14] for the vertical component remain valid. The horizontal components, on the other hand, are not well approximated by the direct emission or the Fresnel approximation (direct + reflected emission), and the surface wave is needed to produce an accurate amplitude and polarization.

The exact calculation also shows that, although the surface wave is a non-radiative field, the exact SDP amplitude falls with the inverse of the distance to the shower core, as pure radiation field would do. The arrival time of the SDP maximum is proportional to the distance to the shower core as well, with a velocity of propagation speed slightly inferior to the speed of light in air, since the surface wave propagates near the boundary and not through the direct path joining emitter and observer.

We must point out that while the present work provides a way of calculating the electric field of a track when a planar boundary is present, we have not discussed the voltage this field would induce in an antenna. Antennas are well understood when working in the far-field regime, with radiating sources. When the sources are near and the field is a mixture of radiating and non-radiating components the reception patterns become more complicated. A proper understanding of the antenna response in the near-field regime of an EAS is capital for a correct prediction of the final voltage.

\section{ACKNOWLEDGMENTS}

We thank the Région Pays de la Loire for its financial support of the Groupe Astro of Subatech and in particular for its contribution to the EXTASIS experiment.

\section{APPENDIX: OBTAINING THE HORIZONTAL DIRECT FIELD FROM THE VERTICAL FIELD}

The direct field created by the horizontal dipole can be obtained from the direct field emitted by the vertical dipole with the appropriate coordinate transformations. This stems from the fact that the direct field is precisely the field calculated as if there were no boundary. Starting with Eq. (12), let us make $z^{\prime}=0$ and place an observer at $(x, 0, z)$, so that $E_{\rho}=E_{x}$ and $E_{y}=0$, without loss of generality. Then, we make the following change of coordinates-we rotate $-\pi$ along the $y$ axis, so that the dipole is oriented towards the $+\hat{x}$ unit vector in the new frame:

$$
\begin{aligned}
& x^{\prime}=z \\
& y^{\prime}=y \\
& z^{\prime}=-x .
\end{aligned}
$$

This transformation (note that in this case $z^{\prime}$ denotes the new $z$ coordinate and not the dipole height) must be applied to the electric field as well:

$$
\begin{aligned}
& E_{x^{\prime}}=E_{z} \\
& E_{y^{\prime}}=E_{y} \\
& E_{z^{\prime}}=-E_{x} .
\end{aligned}
$$

With the observer at $(x, 0, z)$, Eq. (12) changes to the form:

$$
\begin{aligned}
& E_{x}^{d}=-\frac{\omega \mu_{0}}{4 \pi k_{1}^{2}} e^{i k_{1} r_{1}}\left(\frac{i k_{1}^{2}}{r_{1}}-\frac{3 k_{1}}{r_{1}^{2}}-\frac{3 i}{r_{1}^{3}}\right)\left(\frac{x}{r_{1}}\right)\left(\frac{z}{r_{1}}\right) \\
& E_{z}^{d}=\frac{\omega \mu_{0}}{4 \pi k_{1}^{2}} e^{i k_{1} r_{1}}\left[\frac{i k_{1}^{2}}{r_{1}}-\frac{k_{1}}{r_{1}^{2}}-\frac{i}{r_{1}^{3}}-\left(\frac{z}{r_{1}}\right)^{2}\left(\frac{i k_{1}^{2}}{r_{1}}-\frac{3 k_{1}}{r_{1}^{2}}-\frac{3 i}{r_{1}^{3}}\right)\right] .
\end{aligned}
$$

Applying Eqs. (A1) and (A2), along with the identity $x^{\prime 2}=r_{1}^{2}-z^{\prime 2}$, we arrive at

$$
\begin{aligned}
& E_{x^{\prime}}^{d}=\frac{\omega \mu_{0}}{4 \pi k_{1}^{2}} e^{i k_{1} r_{1}}\left[\frac{2 k_{1}}{r_{1}^{2}}+\frac{2 i}{r_{1}^{3}}+\frac{\left(z^{\prime}\right)^{2}}{r_{1}^{2}}\left(\frac{i k_{1}^{2}}{r_{1}}-\frac{3 k_{1}}{r_{1}^{2}}-\frac{3 i}{r_{1}^{3}}\right)\right] \\
& E_{z^{\prime}}^{d}=-\frac{i \omega \mu_{0}}{4 \pi k_{1}^{2}} e^{i k_{1} r_{1}}\left(\frac{x^{\prime}}{r_{1}}\right)\left(\frac{z^{\prime}}{r_{1}}\right)\left(\frac{k_{1}^{2}}{r_{1}}+\frac{3 i k_{1}}{r_{1}^{2}}-\frac{3}{r_{1}^{3}}\right),
\end{aligned}
$$

which is the particular case of Eq. (20) when the observer is located at $(x, 0, z)$ and the dipole is at the origin. We now perform a rotation of an angle $\alpha$ around the $x^{\prime}$ axis. Using the fact that $y^{\prime}=0$ and $E_{y^{\prime}}=0$, the transformations are:

$$
\begin{aligned}
& x^{\prime \prime}=x^{\prime} \\
& y^{\prime \prime}=\sin \alpha z^{\prime} \\
& z^{\prime \prime}=\cos \alpha z^{\prime}
\end{aligned}
$$

and

$$
\begin{aligned}
& E_{x^{\prime \prime}}=E_{x^{\prime}} \\
& E_{y^{\prime \prime}}=\sin \alpha E_{z^{\prime}} \\
& E_{z^{\prime \prime}}=\cos \alpha E_{z^{\prime}} .
\end{aligned}
$$


Let us begin with $E_{z^{\prime \prime}}$. Equations (A5) and (A6) imply that

$$
\begin{aligned}
E_{z^{\prime \prime}}^{d} & =\cos \alpha E_{z^{\prime}}^{d} \\
& =-\cos \alpha \frac{i \omega \mu_{0}}{4 \pi k_{1}^{2}} e^{i k_{1} r_{1}}\left(\frac{x^{\prime \prime}}{r_{1}}\right)\left(\frac{z^{\prime \prime}}{\cos \alpha r_{1}}\right)\left(\frac{k_{1}^{2}}{r_{1}}+\frac{3 i k_{1}}{r_{1}^{2}}-\frac{3}{r_{1}^{3}}\right),
\end{aligned}
$$

and using cylindrical coordinates $\left(\rho, \varphi, z^{\prime \prime}\right)$ we have that $x^{\prime \prime}=\rho \cos \varphi$ and therefore:

$$
E_{z^{\prime \prime}}^{d}=-\frac{i \omega \mu_{0}}{4 \pi k_{1}^{2}} e^{i k_{1} r_{1}} \cos \varphi\left(\frac{\rho}{r_{1}}\right)\left(\frac{z^{\prime \prime}}{r_{1}}\right)\left(\frac{k_{1}^{2}}{r_{1}}+\frac{3 i k_{1}}{r_{1}^{2}}-\frac{3}{r_{1}^{3}}\right),
$$

which is the same as the $z$ component in Eq. (20) if we drop the two primes from the $z^{\prime \prime}$. On the other hand, $E_{x^{\prime \prime}}$ and $E_{y^{\prime \prime}}$ can be written as:

$$
\begin{aligned}
E_{x^{\prime \prime}}^{d} & =E_{x^{\prime}}^{d} \\
& =\frac{\omega \mu_{0}}{4 \pi k_{1}^{2}} e^{i k_{1} r_{1}}\left[\frac{2 k_{1}}{r_{1}^{2}}+\frac{2 i}{r_{1}^{3}}+\frac{\left(z^{\prime \prime}\right)^{2}}{\cos ^{2} \alpha r_{1}^{2}}\left(\frac{i k_{1}^{2}}{r_{1}}-\frac{3 k_{1}}{r_{1}^{2}}-\frac{3 i}{r_{1}^{3}}\right)\right] \\
E_{y^{\prime \prime}}^{d} & =\sin \alpha E_{z^{\prime}}^{d} \\
& =-\frac{\sin \alpha}{\cos \alpha} \frac{i \omega \mu_{0}}{4 \pi k_{1}^{2}} e^{i k_{1} r_{1}}\left(\frac{\rho \cos \varphi}{r_{1}}\right)\left(\frac{z^{\prime \prime}}{r_{1}}\right)\left(\frac{k_{1}^{2}}{r_{1}}+\frac{3 i k_{1}}{r_{1}^{2}}-\frac{3}{r_{1}^{3}}\right) .
\end{aligned}
$$

The sines and cosines of $\varphi$ can be expressed in the following way:

$$
\begin{gathered}
\cos \varphi=\frac{x^{\prime \prime}}{\rho} \\
\sin \varphi=\frac{y^{\prime \prime}}{\rho}=\frac{\sin \alpha z^{\prime}}{\rho}=\frac{\sin \alpha z^{\prime \prime}}{\cos \alpha \rho},
\end{gathered}
$$

from which we derivate the identity:

$$
\sin \varphi \frac{\sin \alpha}{\cos \alpha} z^{\prime \prime}=\frac{\sin ^{2} \alpha}{\rho} \frac{z^{\prime \prime 2}}{\cos ^{2} \alpha} .
$$

We know that the radial field can be obtained by combining the horizontal fields:

$$
E_{\rho}=E_{x^{\prime \prime}} \cos \varphi+E_{y^{\prime \prime}} \sin \varphi,
$$

which, in conjunction with Eqs. (A9), (A10) and (A13) gives:

$E_{\rho}^{d}=\frac{\omega \mu_{0}}{4 \pi k_{1}^{2}} \cos \varphi e^{i k_{1} r_{1}}\left[\frac{2 k_{1}}{r_{1}^{2}}+\frac{2 i}{r_{1}^{3}}+\frac{\left(z^{\prime \prime}\right)^{2}}{r_{1}^{2}}\left(\frac{i k_{1}^{2}}{r_{1}}-\frac{3 k_{1}}{r_{1}^{2}}-\frac{3 i}{r_{1}^{3}}\right)\right]$,

which is consistent with Eq. (20). Finally, expressing the azimuthal field as

$$
E_{\phi}=-E_{x^{\prime \prime}} \sin \varphi+E_{y^{\prime \prime}} \cos \varphi,
$$

along with the following identity:

$$
\begin{aligned}
\frac{\sin \varphi z^{\prime \prime 2}}{r_{1}^{2} \cos ^{2} \alpha}+\cos ^{2} \varphi \frac{\sin \alpha}{\cos \alpha} \frac{\rho z^{\prime \prime}}{r_{1}^{2}} & =\sin \varphi \frac{z^{\prime \prime 2}}{r_{1}^{2}}+\frac{\sin \alpha \rho z^{\prime}}{r_{1}^{2}} \\
& =\frac{\sin \varphi}{r_{1}^{2}}\left(\rho^{2}+z^{\prime \prime 2}\right) \\
& =\sin \varphi,
\end{aligned}
$$

leads us to the final expression for the azimuthal field:

$$
E_{\varphi}^{d}=-\frac{\omega \mu_{0}}{4 \pi k_{1}^{2}} e^{i k_{1} r_{1}} \sin \varphi\left[\frac{i k_{1}^{2}}{r_{1}}-\frac{k_{1}}{r_{1}}-\frac{i}{r_{1}^{3}}\right]
$$

which is the same as in Eq. (20). Equations (A8), (A15) and (A18) show that the direct field of a horizontal dipole can be obtained from the field of a vertical dipole.
[1] F. G. Schröder, Prog. Part. Nucl. Phys. 93, 1 (2017).

[2] S. Buitink et al., Nature (London) 531, 70 (2016).

[3] F. Gaté, B. Revenu, D. García-Fernández, V. Marin, R. Dallier, A. Escudié, and L. Martin, Astropart. Phys. 98, 38 (2018).

[4] CODALEMA collaboration, arXiv:1710.02487v1.

[5] The Pierre Auger Collaboration, Phys. Rev. D 93, 122005 (2016).

[6] P. Schellart et al., Astron. Astrophys. 560, A98 (2013).

[7] W. D. Apel et al., Phys. Lett. B 763, 179 (2016).
[8] S. W. Barwick et al., Astropart. Phys. 90, 50 (2017).

[9] P. Allison et al., Astropart. Phys. 35, 457 (2012).

[10] M. Aartsen et al., arXiv:1412.5106v2.

[11] S. Adrián-Martínez et al., J. Phys. G 43, 084001 (2016).

[12] J. Alvarez-Muñiz et al., arXiv:1810.09994.

[13] V. Marin and B. Revenu, Astropart. Phys. 35, 733 (2012).

[14] D. García-Fernández, B. Revenu, D. Charrier, R. Dallier, A. Escudie, and L. Martin, Phys. Rev. D 97, 103010 (2018).

[15] J. Alvarez-Muñiz, W. R. Carvalho, and E. Zas, Astropart. Phys. 35, 325 (2012). 
[16] T. Huege, M. Ludwig, and C. W. James, AIP Conf. Proc. 1535, 128 (2012).

[17] J. Alvarez-Muñiz, W. R. Carvalho, D. García-Fernández, H. Schoorlemmer, and E. Zas, Astropart. Phys. 66, 31 (2015).

[18] C. A. Balanis, Antenna Theory, Analysis and Design 2nd ed. (John Wiley \& Sons, New York, 1997).

[19] P. Abreu et al., J. Instrum. 7, P10011 (2012).

[20] A. Escudie et al., in Proceedings of the 35th ICRC (PoS, Busan, South Korea, 2017), p. 418.

[21] D. Charrier et al. (to be published).

[22] K. Nishi and K. Suga, in Proceedings of the 20th ICRC (Moscow, Russia, 1987), Vol. 6, p. 125, http://inspirehep .net/record/966153? ln=en.

[23] C. Castagnoli et al., in Proceedings of the 22th ICRC (Dublin, Ireland, 1991), Vol. 4, p. 363, http://inspirehep.net/ record/967518.
[24] B. Revenu et al., in Proceedings 33th ICRC (PoS, Rio de Janeiro, Brazil, 2013), p. 0398.

[25] V. Marin and B. Revenu, arXiv:1211.3305v1.

[26] P. Motloch, J. Alvarez-Muñiz, P. Privitera, and E. Zas, Phys. Rev. D 93, 043010 (2016).

[27] C. W. James, H. Falcke, T. Huege, and M. Ludwig, Phys. Rev. E 84, 056602 (2011).

[28] E. Zas, F. Halzen, and T. Stanev, Phys. Rev. D 45, 362 (1992).

[29] R. King, M. Owens, and T. Wu, Lateral Electromagnetic Waves (Springer-Verlag, New York, 1992).

[30] K. A. Michalski, IEEE Trans. Antennas Propag. 46, 1405 (1998).

[31] D. García-Fernández, J. Alvarez-Muñiz, W. R. Carvalho, Jr., A. Romero-Wolf, and E. Zas, Phys. Rev. D 87, 023003 (2013).

[32] A. Aab et al., J. Instrum. 12, T10005 (2017).

[33] Recommendation ITU-R P.527-3, https://www.itu.int/rec/RREC-P.527-3-199203-S/en. 